\title{
Sodium Radiofrequency Coils for Magnetic Resonance: From Design to Applications
}

\author{
Giulio Giovannetti ${ }^{1,2}, *(\mathbb{D})$, Alessandra Flori ${ }^{3}$, Nicola Martini ${ }^{3}\left(\mathbb{D}\right.$, Roberto Francischello ${ }^{3,4}(\mathbb{D}$, \\ Giovanni Donato Aquaro ${ }^{2}$, Alessandro Pingitore ${ }^{1}\left(\mathbb{D}\right.$ and Francesca Frijia ${ }^{3}$ \\ 1 Institute of Clinical Physiology, National Research Council (CNR), 56124 Pisa, Italy; pingi@ifc.cnr.it \\ 2 Fondazione CNR/Regione Toscana G. Monasterio, 56124 Pisa, Italy; aquaro@ftgm.it \\ 3 U.O.C. Bioengineering and Clinical Technology, Fondazione CNR/Regione Toscana G. Monasterio, \\ 56124 Pisa, Italy; alessandra.flori@ftgm.it (A.F.); nicola.martini@ftgm.it (N.M.); \\ roberto.francischello@gmail.com (R.F.); f.frijia@ftgm.it (F.F.) \\ 4 Dipartimento di Chimica e Chimica Industriale, Università di Pisa, 56124 Pisa, Italy \\ * Correspondence: giovannetti@ifc.cnr.it
}

check for updates

Citation: Giovannetti, G.; Flori, A.; Martini, N.; Francischello, R.; Aquaro, G.D.; Pingitore, A.; Frijia, F. Sodium Radiofrequency Coils for Magnetic Resonance: From Design to Applications. Electronics 2021, 10, 1788. https://doi.org/10.3390/ electronics10151788

Academic Editors: Youn Tae Kim, Junghwan Hwang and Meina Li

Received: 29 June 2021

Accepted: 21 July 2021

Published: 26 July 2021

Publisher's Note: MDPI stays neutral with regard to jurisdictional claims in published maps and institutional affiliations.

Copyright: (c) 2021 by the authors. Licensee MDPI, Basel, Switzerland. This article is an open access article distributed under the terms and conditions of the Creative Commons Attribution (CC BY) license (https:// creativecommons.org/licenses/by/ $4.0 /)$.

\begin{abstract}
Sodium $\left({ }^{23} \mathrm{Na}\right)$ is the most abundant cation present in the human body and is involved in a large number of vital body functions. In the last few years, the interest in Sodium Magnetic Resonance Imaging $\left({ }^{23} \mathrm{Na} \mathrm{MRI}\right)$ has considerably increased for its relevance in physiological and physiopathological aspects. Indeed, sodium MRI offers the possibility to extend the anatomical imaging information by providing additional and complementary information on physiology and cellular metabolism with the heteronuclear Magnetic Resonance Spectroscopy (MRS). Constraints are the rapidly decaying of sodium signal, the sensitivity lack due to the low sodium concentration versus ${ }^{1} \mathrm{H}$-MRI induce scan times not clinically acceptable and it also constitutes a challenge for sodium MRI. With the available magnetic fields for clinical MRI scanners (1.5 T, $3 \mathrm{~T}, 7 \mathrm{~T})$, and the hardware capabilities such as strong gradient strengths with high slew rates and new dedicated radiofrequency (RF) sodium coils, it is possible to reach reasonable measurement times ( 10-15 $\mathrm{min})$ with a resolution of a few millimeters, where it has already been applied in vivo in many human organs such as the brain, cartilage, kidneys, heart, as well as in muscle and the breast. In this work, we review the different geometries and setup of sodium coils described in the available literature for different in vivo applications in human organs with clinical MR scanners, by providing details of the design, modeling and construction of the coils.
\end{abstract}

Keywords: magnetic resonance; sodium imaging; radio frequency coils

\section{Introduction}

In recent years, the scientific interest in sodium $\left({ }^{23} \mathrm{Na}\right)$ magnetic resonance imaging (MRI) and spectroscopy (MRS) has experienced a significant increase.

While hydrogen $\left({ }^{1} \mathrm{H}\right) \mathrm{MRI}$ is an established non-invasive diagnostic technique for investigating the morpho-functional status of a tissue, MRS of both hydrogen and nuclei other than hydrogen (so-called heteronuclei) can derive complementary biochemical information on tissue physiology and viability, as well as on cellular metabolism.

Among the heteronuclei that can be investigated by magnetic resonance approaches, sodium has a prominent role because of its pathophysiological relevance.

Sodium is the most abundant cation in the human body, where it is involved in maintaining the homeostasis of the organism and in other vital body functions. In particular, the sodium transmembrane electrochemical gradient is implicated in the generation and propagation of the action potentials responsible for several physiological functions, such as transmission of nerve impulses, muscle contractions and cardiac activity. Moreover, sodium significantly contributes to the maintenance of the resting cell membrane potential, 
mainly through the action of the $\mathrm{Na}+/ \mathrm{K}+$-ATPase pump (i.e., the sodium-potassium pump) [1].

Sodium concentration depends on the tissue metabolic state and is strictly related to the integrity of the cell membrane. Disruption of the cellular membrane in pathologic tissue modifies the large sodium concentration gradient between the intracellular and extracellular compartments, resulting in increased intracellular sodium. Sodium accumulation has been thus reported in different pathologic conditions such as tumors [2,3], ischemia [4,5] and neurodegenerative diseases like multiple sclerosis [6,7] or Alzheimer's Disease [8].

Sodium MRI and MRS exploit the signal derived from endogenous sodium nuclei (i.e., without any contrast media injection) and the signal intensity is directly determined by the sodium tissue content. Therefore, sodium MRI/MRS gained interest as non-invasive techniques for the quantification of tissue sodium concentration. In particular, by detecting alterations in the total tissue sodium concentration, sodium MRI/MRS approaches can discriminate between viable and non-viable tissue $[9,10]$. Tissue sodium concentration is thus increasingly recognized as a sodium MRI-derived biomarker for the investigation of cell function and viability. In this regard, methods for the differentiation of intra- and extracellular sodium have been proposed [11-13].

From the point of view of the magnetic properties, sodium is a spin $3 / 2$ quadrupolar nucleus generating the second strongest nuclear magnetic resonance signal after hydrogen. However, mainly due to the low in vivo concentration (10-15 mM intracellular, $140-150 \mathrm{mM}$ extracellular concentrations, respectively), sodium MRI is characterized by a low average signal-to-noise ratio (SNR) and limited sensitivity compared to hydrogen $[9,14]$.

Due to the low biological concentration, the fast transverse relaxation rate and lower gyromagnetic ratio $\left(\gamma_{\mathrm{Na}}=11.26 \mathrm{MHz} / \mathrm{T}\right.$, approximately one-quarter of that of hydrogen at $\left.\gamma_{\mathrm{H}}=42.57 \mathrm{MHz} / \mathrm{T}\right)$, achieving high spatial resolution at acceptable SNR is challenging in sodium MRI/MRS studies.

Addressing the limited sensitivity of sodium MRI places significantly increased demands on the gradient hardware, not typically available on clinical scanners, to achieve the desired resolution and minimize the readout duration.

Finally, the lower Larmor's frequency of sodium requires custom radiofrequency (RF) hardware, as broadband RF amplifier, custom RF transmit and receive coil, sodiumfrequency low-noise pre-amplifier, and transmit/receive $(\mathrm{Tx} / \mathrm{Rx})$ circuitry.

In particular, the design and development of dedicated RF coils are necessary constraints for maximizing SNR in sodium experiments [15].

For obtaining high-quality MR data, RF coils should be able to support large fieldof-view (FOV) with high RF magnetic field homogeneity in transmission and to achieve high SNR in reception. Moreover, the co-registration of sodium data with hydrogen images requires that the MR system can efficiently operate at two different frequencies.

With the increasing magnetic field strength of modern MRI scanners (1.5 T, $3 \mathrm{~T}, 7 \mathrm{~T})$, the improved hardware capabilities such as strong gradient strengths with high slew rates, and new dedicated RF sodium coils, it is now possible to reach reasonable measurement times ( 10 min-15 min) with a resolution of a few millimeters. Thanks to the recent developments, sodium MRI has been applied for the in vivo investigation of many human organs such as the brain, cartilage, kidneys, heart, as well as for muscle and breast $[1,10]$.

In this work, we review different geometries and setup of sodium- dedicated RF coils. In particular, Section 2 briefly describes the RF coil theory in general, comprising details for the design, modelling, construction and test of the coils, while Section 3 analyzes the sodium coil typologies (surface, volume and phased-array coils) described in literature for different in vivo applications in human organs by using clinical MR scanners. Section 4 is dedicated to the different pulse sequences and image reconstruction methods for in vivo application, and Section 5 contains final considerations related to clinical applications of sodium MR. 


\section{Coil Theory}

\subsection{Coil Design}

Sodium MRI can be performed on commercial MRI scanners equipped with a broadband $x$-nuclei RF amplifier for transmission and reception. The sodium coils are connected to the RF system using an interface box with transmit/receive switches and integrated low-noise preamplifiers.

The $B_{1}$ field is generated and picked-up by transmitter (Tx) and receiver (Rx) coils, respectively [16]. The transmitter coil has to produce a highly homogeneous field in the desired field of view (FOV) and to achieve this, it is usually larger in size to optimize the magnetic field homogeneity and include a large volume of tissue. The receiver coil function is to maximize the signal detection and, in the same time, minimizing the noise, therefore it is optimal to minimize its dimensions. In general, both transmit and receive coils must be adapted to the specific application and to the sample size. Nonetheless, they have to keep good performances with slightly different sample geometries.

MR coils are categorized into volume, surface and phased-array coils, according to their shapes [17]. Volume coils are often used both for transmission and reception and generate a uniform magnetic field in a large region surrounding the sample. Surface coils are constituted by loops of various shapes and are much smaller than the volume coils, providing higher SNR, but with relatively poor magnetic field homogeneity [16]. Phased-array coils [18], whose individual element is generally constituted by circular or rectangular loops, permits high SNR with a large region of sensitivity. Such coils permit to reduce imaging time thanks to their ability to spatially localize signals and their design is a practical trade-off between maximizing SNR by using close-fitting arrays and optimizing patient comfort and clinical workflow. The single element for use in the array has to be optimized for adjusting the sensitivity pattern of the entire array and at the same time for minimizing crosstalk between elements. Moreover, phased-array coils enable parallel imaging, which is a technique that may provide substantial reductions in image acquisition time by using coil geometric factors [19].

Linear coils produce or pick up only a linear component of the circular polarized RF field, while the use of quadrature coils increases the SNR by a factor of up to $\sqrt{ } 2$ by producing two orthogonal RF fields (circular polarization) and decreasing the power by a factor of 2, resulting in a lower specific absorption rate (SAR) [20].

Finally, for the integration of sodium with hydrogen imaging, the MR system must operate at two different frequencies and this can be achieved with two isolated coils or by using a dual-tuned coil (DT). The latter have the great advantage of simplifying patient setup and performing anatomical localization and sodium data collection in sequence without the need of patient repositioning.

In coils for MR, the flowing current is maximal at the Larmor's frequency $\left(f_{0}=\gamma / 2 \pi\right.$ $B_{0}$, where $B_{0}$ is the static field and $\gamma$ is the gyromagnetic ratio for sodium nucleus) which corresponds to the coil's resonance frequency described by Equation (1):

$$
f_{0}=\frac{1}{2 \pi \sqrt{L C}}
$$

where $L$ inductance takes into account the energy stored in the magnetic field and $C$ capacitance mainly results from the discrete capacitors contribution. The current flowing in the coil is mainly limited by loss mechanisms (schematized with a $R$ total resistance) which take into account the conductor losses $\left(R_{\text {coil }}\right.$ resistance), sample losses $\left(R_{\text {sample }}\right.$ resistance) and radiative and tuning capacitor losses ( $R_{\text {extra }}$ resistance). This RLC circuit is characterized by a $Q$ quality factor as defined in Equation (2) [16]:

$$
Q=\frac{2 \pi f_{0} L}{R}=\frac{1}{R} \sqrt{\frac{L}{C}}
$$


and by the ratio $r$ between the empty resonator quality factor $\left(Q_{\text {unloaded }}\right)$ and the one with the resonator loaded with the sample $\left(Q_{\text {loaded }}\right)$, as described in the Equation (3):

$$
r=\frac{Q_{\text {unloaded }}}{Q_{\text {loaded }}}=1+\frac{R_{\text {sample }}}{R_{\text {coil }}+R_{\text {extra }}}
$$

Typical values range from 50 to 600 for $Q$ and from 2 to 6 for $r$. This last value assures the minimization of the losses in the coil and a consequent SNR improvement [19].

The $L$ inductance depends on the conductor size and geometry (strip or wire) and can be calculated by using Equation (4) [16]:

$$
L=\frac{\mu_{0}}{4 \pi I^{2}} \iiint_{V} \iiint_{V} \frac{J(r) \cdot J\left(r^{\prime}\right)}{R} d v d v^{\prime}
$$

where $J$ is the current density in the conductor, $\mu_{0}$ is the permeability of free space, $I$ represents the total current in the conductor, $V$ is the conductor volume and $R=\left|r-r^{\prime}\right|$.

The magnetic field homogeneity is another important parameter in the coil design, since the FOV depends on it [18].

\subsection{Coil Simulation}

In order to estimate the coils magnetic field pattern, two different approaches can be employed. The first one is based on magnetostatic theory, which implies the assumption of a nearly static field holding for coils whose dimensions are much lower than the wavelength. Such approach has been demonstrated useful for the design and simulation of low frequency-tuned coils constituted by circular and linear conductor segments [21]. The magnetic field calculation can be performed by subdividing the coil conductor in small segments for separately studying their total magnetic field contribution. The calculation of the three-dimensional magnetic field pattern produced by the current flowing in a coil can be performed with Biot-Savart law [16] by neglecting the conductor sizes with respect to the wavelength and by considering the coil conductor as a very thin wire. When the coil tuning frequency increases, RF fields interact more strongly with the sample and the magnetostatic approach is not more suitable for coil design and simulation. In this case, different electromagnetic-numerical methods based on solutions to Maxwell's equations can be employed for the magnetic field calculation, including the Finite-Difference Time-Domain (FDTD), the Finite Element Methods (FEM) and the Method of Moments (MoM) [22].

\subsection{Coil Test}

After simulation and prototype implementation, the work bench testing of the MR coils was carried out in order to evaluate their performance. A practical way to perform $Q$ coil quality factor measurement is based on Equation (5):

$$
Q=\frac{f_{0}}{B}
$$

where $B$ is the $-3 \mathrm{~dB}$ coil bandwidth.

Equation (5) can be used for the measurement of both loaded and unloaded coil quality factors. Such measurements can be easily performed by using a dual-loop probe, consisting of two pickup loops partially overlapped to minimize the mutual coupling between the elements, and a network analyzer. Figure 1 summarizes the simulation, design and test phases for a RF coil. 

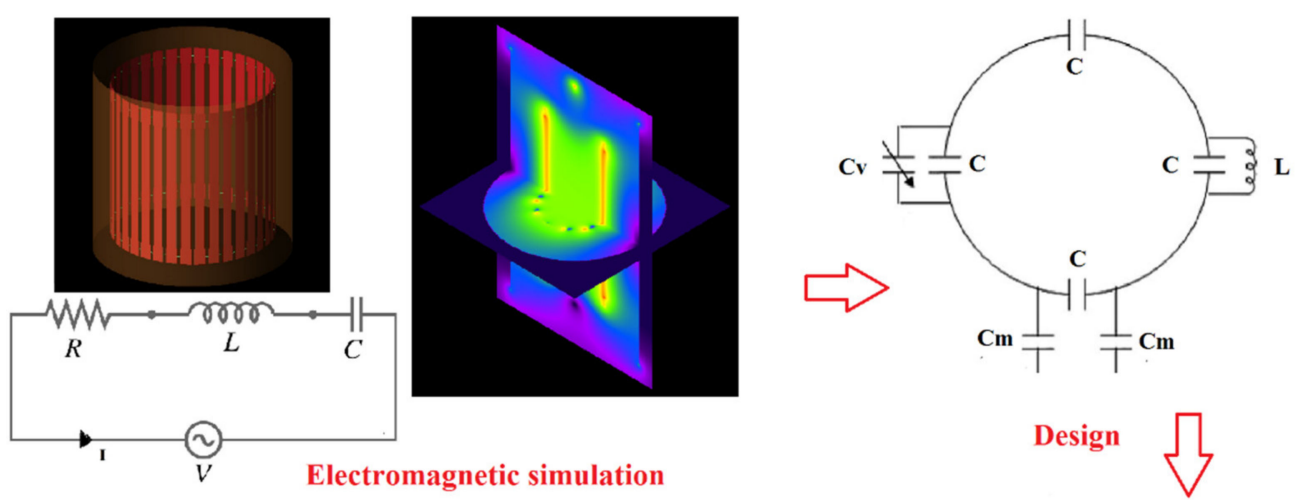
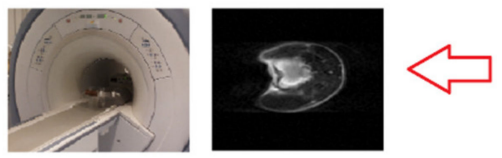

MR image acquisition

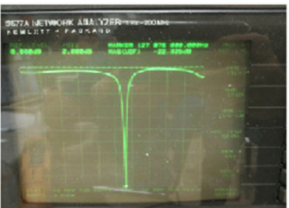

Workbench test

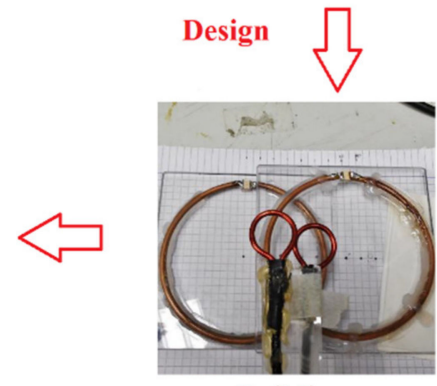

Building

Figure 1. The different phases in the RF coil development.

\section{Sodium Coils}

\subsection{Surface and Volume Sodium-Only Coils}

A study conducted for determining the sodium MRI potential for a $0.5 \mathrm{~T}$ field MRI scanner Bruker Tomikon S50 employed the standard transmit/receive (T/R) switch and a custom coil [23].

The coil is a $5.58 \mathrm{MHz}$-tuned $20 \mathrm{~cm}$ square-shaped Tx/Rx coil constituted by 4 turns of $0.5-\mathrm{mm}$ copper wire, connected to the scanner $\mathrm{T} / \mathrm{R}$ switch with a $3.8 \mathrm{~m}$-long RF cable.

Such a whole-body (WB) coil, depicted in Figure 2, was designed for performing the sodium MRI of different body parts (head, kidneys, liver, heart, gallbladder, stomach and bowel). The unloaded quality factor $Q$ (Equation (2)) and the ratio $r$ (Equation (3)) when the coils were loaded resulted to be, respectively, 280 and 2.3. A second sodium volume birdcage $\mathrm{Tx} / \mathrm{Rx}$ coil was realized by modifying the RF circuit and by adding side extenders to the manufacturer hydrogen receive-only coil originally designed for human extremities study. Such coil permitted to optimize pulse sequences parameters thanks to its higher field homogeneity respect to the sodium WB coil and was used for in vivo human head sodium studies (Figure 3).

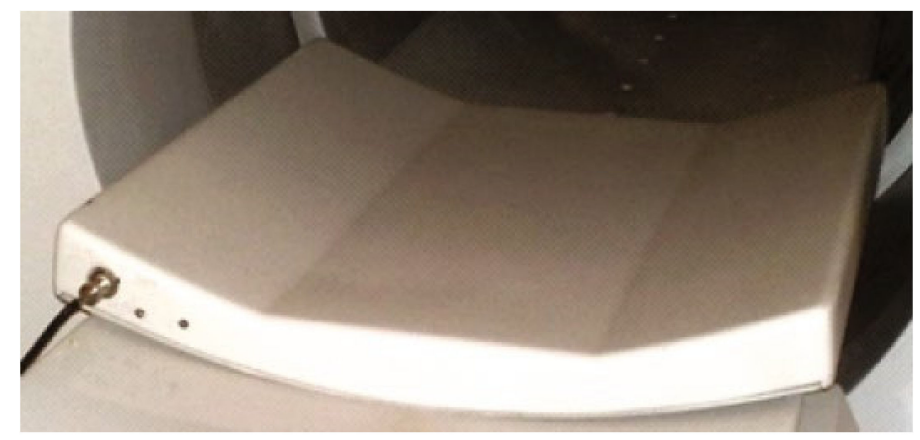

Figure 2. Sodium Whole Body coil. Reprinted by permission from Anisimov et al., App Magn Reson 2019 [23]. 


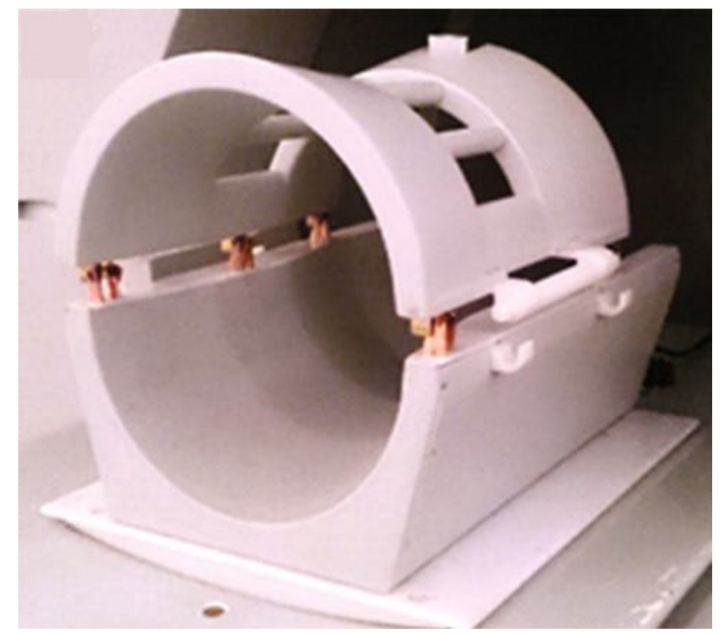

Figure 3. Sodium transceiver birdcage coil. Reprinted by permission from Anisimov et al., App Magn Reson 2019 [23].

Although sodium WB images in prone and supine positions were obtained only in coronal plane due to the limited penetration depth of the RF field generated by such surface coil, a good registration of sodium signal corresponding to proper anatomy was obtained. It resulted in the high similarity of the sodium signal distribution graphs along the human body and the body volume (mass). Figure 4 shows hydrogen and sodium images obtained from the subject. In particular, Figure 4a shows the image obtained by summing up 12 individual slices obtained from multi-slice Gradient echo (GRE) scan, while image of Figure $4 \mathrm{~b}$ is Maximum Intensity Projection (MIP) obtained using Fast Spin Echo-Short-TI Inversion Recovery (FSE-STIR).
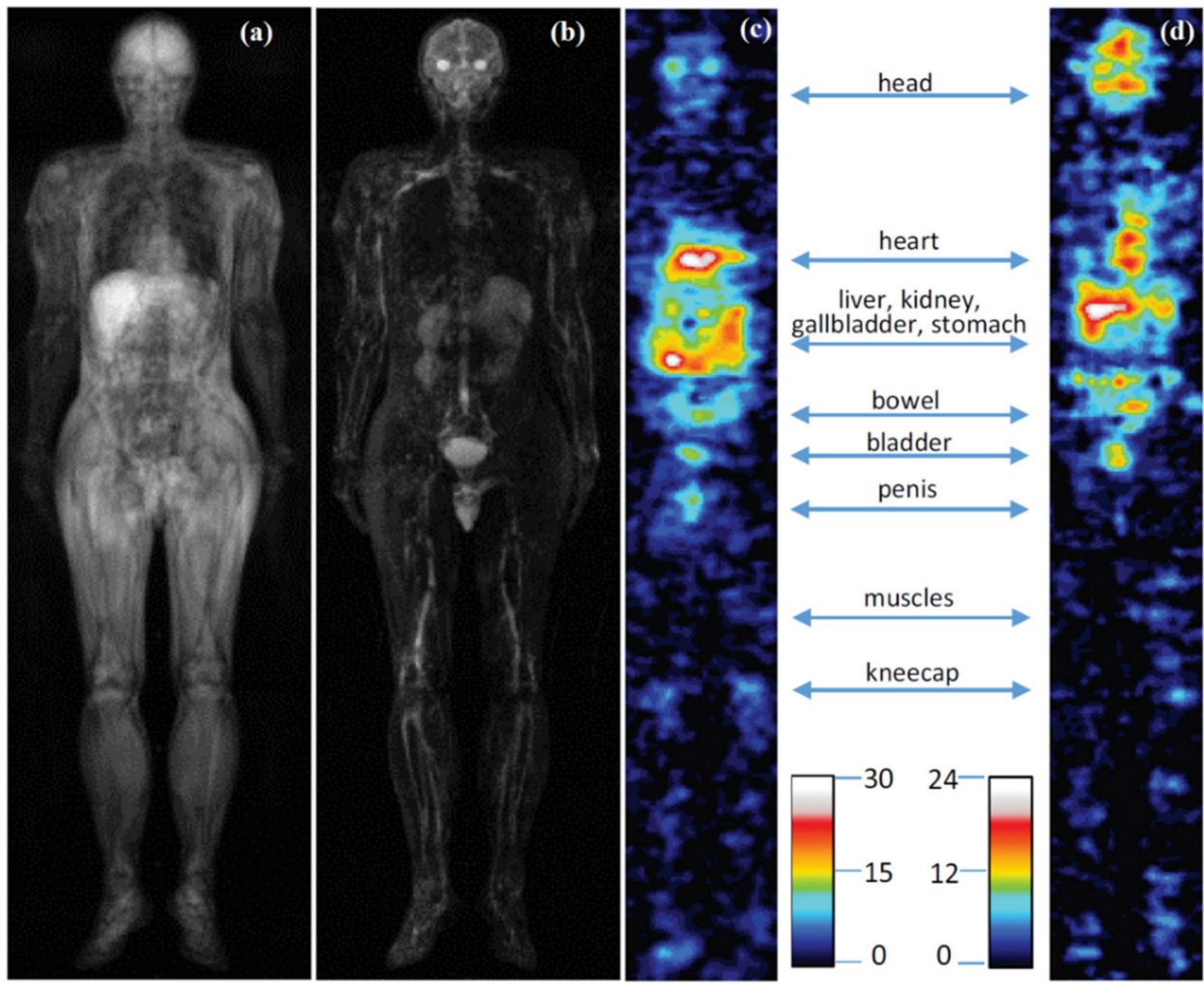

Figure 4. (a) Hydrogen WB MRI in supine position; (b) sodium WB MRI; (c) SNR maps in prone position; (d) supine positions. Reprinted by permission from Anisimov et al., App Magn Reson 2019 [23]. 
Details about design, simulation, construction and test of a Tx/Rx $25 \mathrm{~cm}$ diameter circular coil designed for sodium MR for phantoms and humans with a clinical $3 \mathrm{~T}$ scanner were described in [24]. For the coil simulation, magnetostatic approach (Biot-Savart law) was employed for 3D magnetic field distributions estimation while the coil inductance calculation was performed by using Equation (4). The coil was built using a thin copper foil (10 mm width and $70 \mu \mathrm{m}$ thickness strip) etched on a printed circuit board (PCB), while the coil tuning and matching were performed with high $Q$-factor capacitors (ATC 100C, American Technical Ceramics, Huntington Station, NY, USA). Finally, a resonant blocking circuit tuned at hydrogen frequency $(127.75 \mathrm{MHz})$ was added for minimizing its interaction with the WB hydrogen coil. Figure 5 shows a sketch of the designed coil, while Figure 6 depicts the magnetic field pattern.

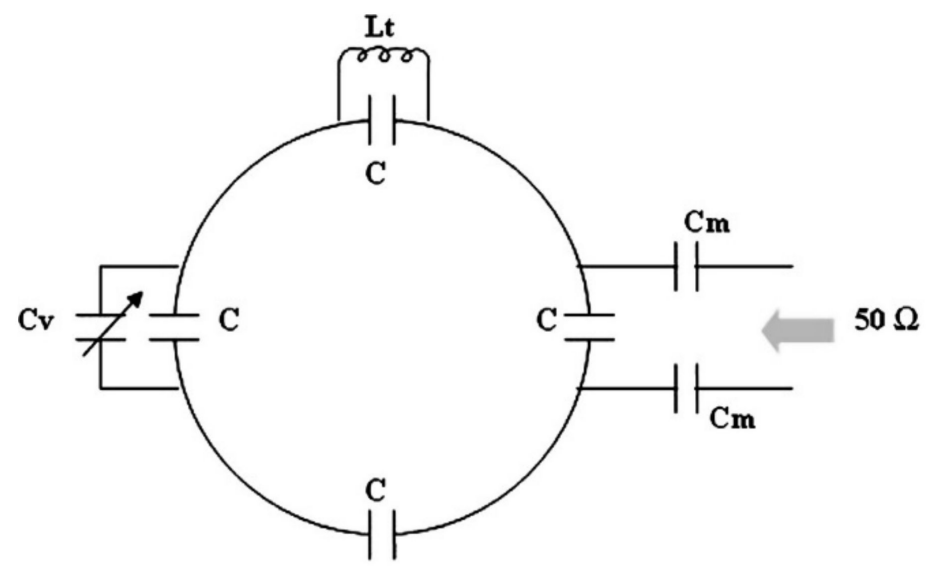

Figure 5. Sketch of the sodium surface coil. Reprinted by permission from Giovannetti et al., Measurement 2014 [24].

Preliminary workbench tests provided unloaded $Q$ and $r$ values of, respectively, 75 and 2.2 for a load constituted by a pair of cubic samples filled with $2.4 \mathrm{~g} / \mathrm{L} \mathrm{NaCl}$. To evaluate the coil sensitivity in discriminating different sodium concentrations, a dedicated phantom filled with distilled deionized water and $\mathrm{NaCl}$ salt at different concentrations $(38,77,154$, 231 and $308 \mathrm{mM}$ ) was built and employed for data acquisitions. Successively calf, brain, kidney and heart images were acquired on healthy volunteers with a 3 T GE Excite HDx (GE HealthCare, Waukesha, WI, USA) scanner including Multinuclear Spectroscopy (MNS) facilities. Sodium Chemical Shift Imaging (CSI) maps were overlapped on anatomical images acquired with the WB coil. Results demonstrated the good performance of the designed coil for sodium quantification in different human organs, thanks to its possibility to obtain high quality data from tissues close to the human body surface.

In [25] the use of a quadrature surface coil for studying the sodium distribution in the human kidney with a 3 T scanner (Signa LX; General Electric, Waukesha, WI, USA) was reported. Such coil, which combined two surface coils into a quadrature structure (Figure 7), was constituted by two loops of $18 \mathrm{~cm}$ side which are overlapped in $20 \%$ of their area for avoiding coupling between them. Successively, the coils were connected to a quadrature combiner and finally to a transmitter/receiver switch and a preamplifier. 


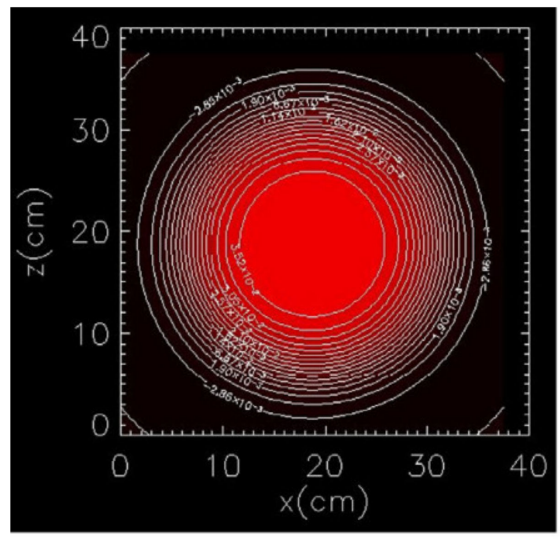

(a)

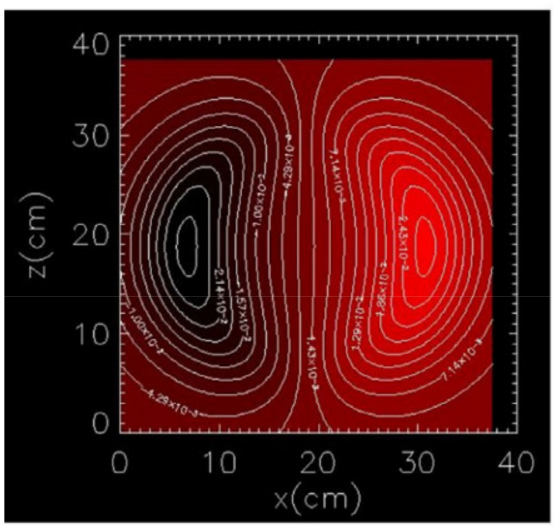

(c)

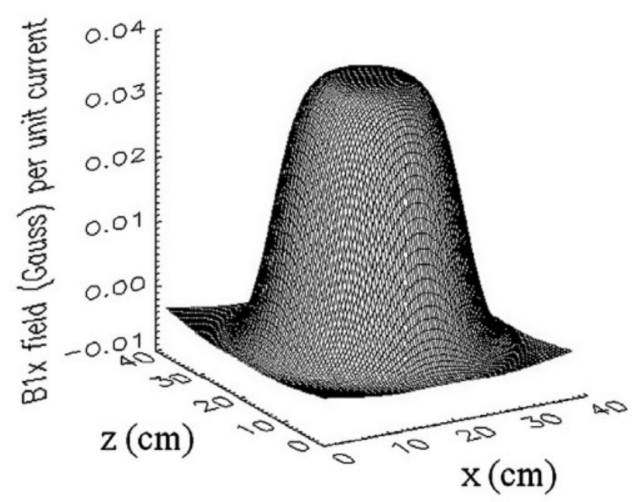

(b)

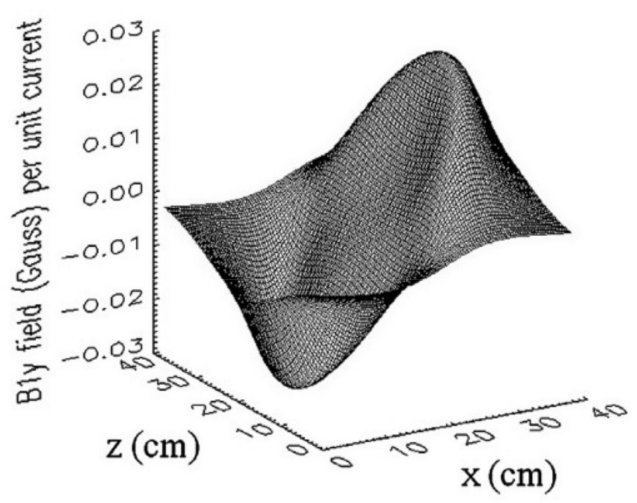

(d)

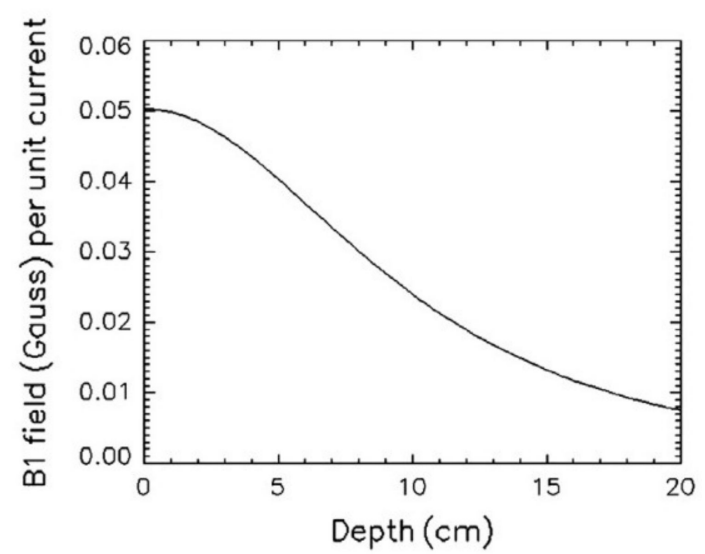

(e)

Figure 6. (a) Coil magnetic field pattern: Bx component as contour plot; (b) 3D plot; (c) by component as contour plot; (d) 3D plot; (e) normalized magnetic field along coil axis. Reprinted by permission from Giovannetti et al., Measurement 2014 [24]. 


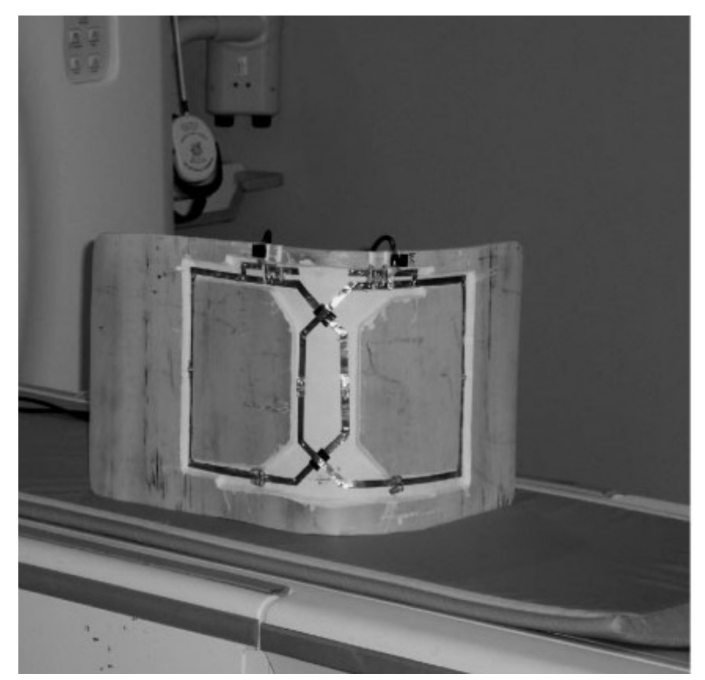

Figure 7. Quadrature surface coil. Reprinted by permission from Maril et al., Magn Reson Med 2006 [25].

The surface coil dimensions were chosen as a function of the distance of the kidney center from the body posterior surface. The designed coil guaranteed an optimal compromise between SNR and coverage of the kidney in the coronal plane. In this work the acquisition of the hydrogen images for localization was performed with the body coil.

\subsection{Surface and Volume Dual-Tuned Sodium/Hydrogen Coils}

The simulation and the design of a dual-tuned sodium/hydrogen surface RF coil for knee MR imaging with an Ultra High Field (UHF) 7 T MRI scanner is described in [26]. The coil was constituted by two concentric loops, with the inner tuned at $78.89 \mathrm{MHz}$ (sodium frequency@ 7 T) and the outer at 298.03 MHz (hydrogen frequency @ 7 T), and both loops were employed as Tx/Rx coils. The decoupling between the two loops was achieved by the insertion of a trap circuit in the sodium loop. Such trap, constituted by a parallel LC resonant circuit, acts as an open circuit at hydrogen frequency. The dual-tuned coil, previously simulated with CST Microwave Studio tool for evaluating magnetic and electric field maps at both tuned frequencies, was tested at workbench by using a cylinder phantom equivalent to the human knee. The hydrogen loop and sodium loop provided a quality factor of 88 and 217, respectively, while the $r$ ratios were 3.8 for the hydrogen loop and 5.9 for the sodium loop. Successively, such coil was employed for in vivo acquisition on healthy volunteers, showing the great advantage of using UHF MRI for the knee cartilage imaging [26].

WB sodium MRI with a clinical $3 \mathrm{~T}$ scanner was performed with an asymmetrical 16 legs quadrature birdcage coil (STARK Contrast, Erlangen, Germany) with $34 \mathrm{~cm}$ height, $47.5 \mathrm{~cm}$ width and $50 \mathrm{~cm}$ length. Such a coil could be used as a Tx/Rx coil or a Tx-only coil in conjunction with a Rx-only surface coil, thanks to the use of an active decoupling circuit incorporated into the coil design [27]. The asymmetric design was chosen for employing the entire available space inside the bore, while maintaining the highest magnetic field homogeneity. The coil was surrounded by an RF shield, necessary for preventing coupling with the nearby gradient coils.

The coil was simulated with FEKO (EM Software and Systems GmbH, Boblingen, Germany) electromagnetic simulation software for evaluating magnetic field homogeneity when the coil was virtually loaded with a phantom whose electromagnetic properties mimicked the human tissue ones. Workbench tests performed with an $0.9 \%$ saline solution homogeneous phantom provided a coil $r$ value of 2.3. 3D whole body sodium MRI performed on a healthy male volunteer demonstrated that calf, brain, prostate, vertebral discs, arms, legs, knees and hands could be resolved with sub-cm spatial resolution and with a scan time of less than $60 \mathrm{~min}$. Moreover, an SNR improvement up to fivefold was 
achieved when using the combination of the Tx birdcage and the local $\mathrm{Rx} 20 \mathrm{~cm}$ diameter circular coil [28].

A description of the design and the development of a dual-tuned hydrogen/sodium Tx/Rx birdcage coil for MR human calf using a 3 T MR scanner was detailed in [29]. The dual-tuned birdcage structure was designed with legs alternate tuning, in order to build two "virtual coils" with different circuital elements for the even and the odd legs for the tuning to hydrogen and sodium frequency, respectively. The decoupling between sodium and hydrogen channels was achieved by inserting hydrogen frequency tuned trap circuits in the sodium-legs, as shown in Figure 8.

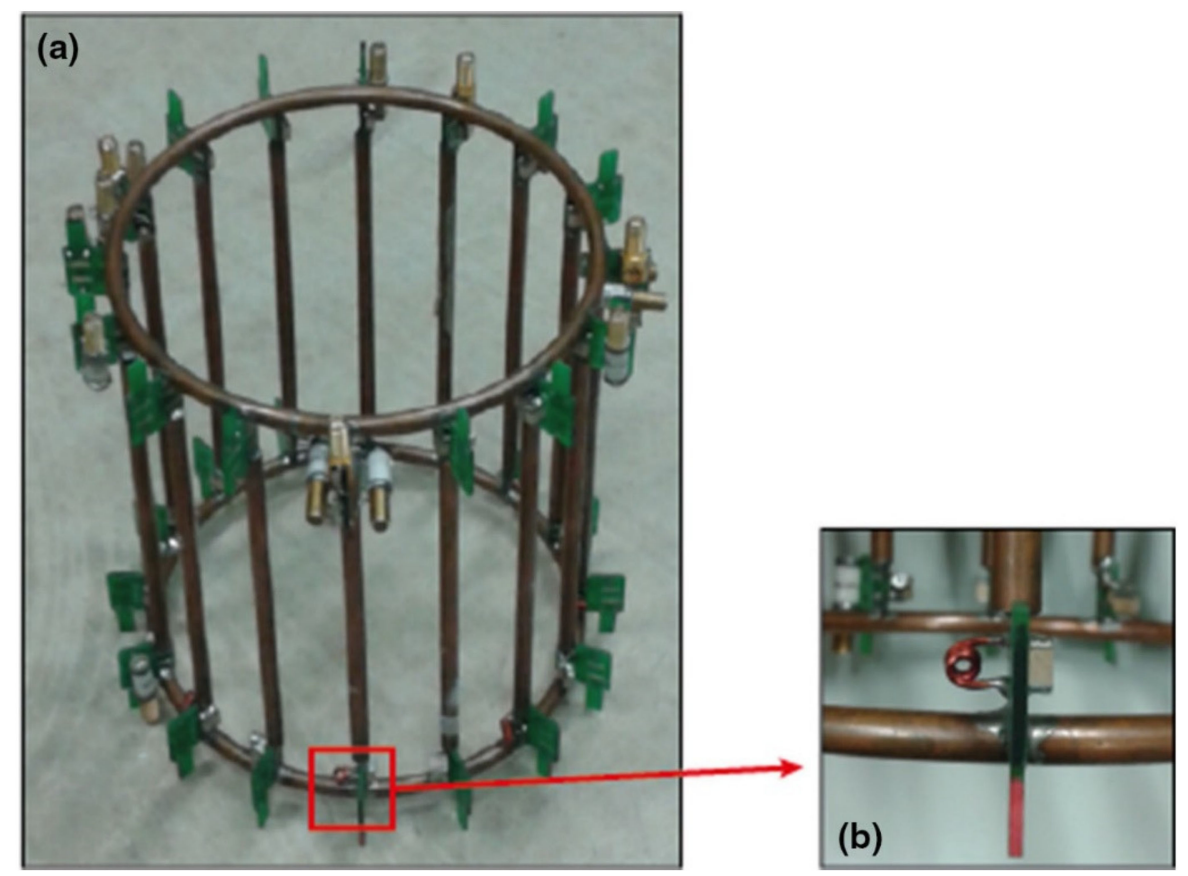

Figure 8. (a) The built birdcage coil; (b) the trap circuit. Reprinted by permission from Giovannetti et al., App Magn Reson 2015 [29].

FDTD numerical simulations performed with a commercial software XFdtd (Remcom, State College, PA, USA) permitted to evaluate the decoupling efficiency and the magnetic field pattern at both tuning frequencies for the coil loaded with a biological phantom.

After implementing the coil prototype and inserting the tuning, matching and decoupling circuits, the workbench tests were performed, in order to measure unloaded $Q$ and $r$ values when the coil was loaded with a cylindrical homogeneous phantom of saline solution ( $55 \mathrm{mM}$ of $\mathrm{NaCl}$ and $5 \mathrm{mM}$ of $\mathrm{NiCl}_{2}$ ). For the hydrogen channel, $Q$ and $r$ resulted 291 and 5.3, respectively, while the sodium channel provided $Q=147$ and $r=2.0$. Moreover, scattering parameter measurements evidenced a correct quadrature polarization of the hydrogen and sodium channels magnetic field and an appropriate decoupling between the two channels. Successively, the performance of the dual-tuned coil was evaluated with phantom (Figure 9) and in vivo experiments on human calves with the clinical MR scanner, permitting to obtain good sodium maps. 

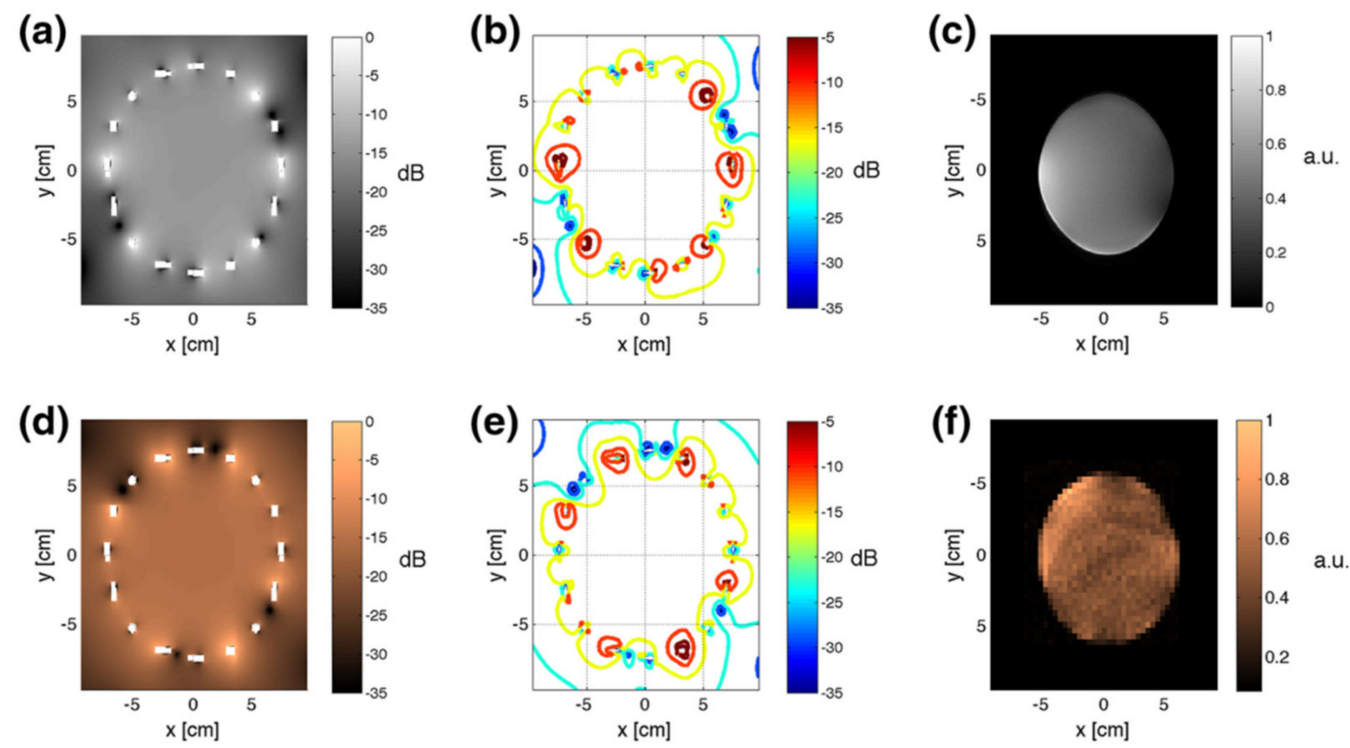

Figure 9. (a) Simulated and measured field maps in the central axial plane: Simulated field map; (b) corresponding contour plot; (c) measured field map at hydrogen frequency; (d) simulated field map; (e) corresponding contour plot; (f) measured field map at sodium frequency. Reprinted by permission from Giovannetti et al., App Magn Reson 2015 [29].

\subsection{Phased-Array Sodium-Only Coils}

Qian [30] built a 15-channel array coil for sodium imaging for the brain at $7 \mathrm{~T}$. The outside part is a cylindrical birdcage coil as transmitter/receiver and can be used as a single-channel volume coil, producing a uniform $B_{1}$ field for excitation over the whole head region. The inside part is an inserted receive-only array coil composed of 15 circular loops arranged in two rows on a helmet-like frame that pick up stronger signals due to their position closer to the head.

The coil performance was tested evaluating the SNR with a phantom and comparing the values with a volume coil. The array coil produced a similar SNR in the central region of the phantom, 1-2-fold SNR in the middle region, and 2-fold SNR in the peripheral region. To improve SNR into deep brain areas the authors applied a noise decorrelation method across channels of the array coil. After the noise decorrelation the SNR resulted extended 2 -fold from the peripheral into the middle region. Moreover, with the 15-channel array coil the SNR was doubled over the volume coil in an average over a lower region of phantom.

The 15-channel array coil showed also a significant improvement in the visualization of in vivo anatomical details in the whole brain applying noise decorrelation method. The in vivo experiments confirm the same results found in phantom.

Robson [31] designed a sodium array coil for heart study with a $3 \mathrm{~T}$ scanner, which consisted of two identical coil halves, each containing a transmit loop and four receive-only channels in a Helmholtz-like configuration. All Rx elements were actively decoupled by a sodium trap whilst the Tx loops contained switched PIN diodes for detuning. Hydrogen traps were added to permit the use of the Tx/Rx hydrogen body coil.

To prove that the array technology improved SNR in sodium imaging of the heart at $3 \mathrm{~T}, \mathrm{Q}$ factor of the Rx and Tx elements were evaluated. The $\mathrm{Q}$ factor for Rx elements dropped from 220 by a factor of 2.8 when loaded physiologically, while the $\mathrm{Q}$ drop of the Tx elements was 5.5. The active decoupling of the Rx elements as well as of the Tx elements resulted better than $-20 \mathrm{~dB}$ and the decoupling of neighboring elements was better than $-20 \mathrm{~dB}$. Figure 10 shows the HLA view of a hydrogen True Fast Imaging with Steady Precession (FISP) acquired with the hydrogen body coil and the corresponding two-dimensional Ultrashort Echo Time (2D-UTE) sodium image. 

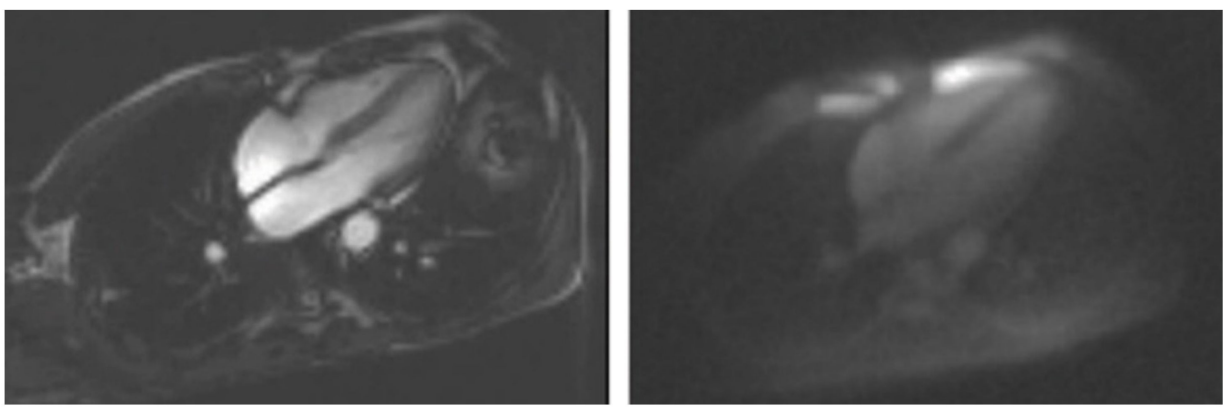

Figure 10. Hydrogen image True-FISP (left) and sodium image 2D-UTE (right). Reprinted by permission from Robson et al., J Cardio Magn Reson 2008 [31].

\subsection{Phased-Array Dual-Tuned Sodium/Hydrogen Coils}

Brown [32] described eight-channel receive-only rectangular array coils for the human knee articular cartilage at $7 \mathrm{~T}$, implemented without standard dual-resonance circuitry to provide improved sodium SNR. A detunable sodium birdcage was added for homogeneous sodium excitation and a four-channel hydrogen transmit-receive array was added to provide anatomical reference imaging and $\mathrm{B}_{0}$ shimming capabilities. Both additional modules were implemented with minimal disturbance to the eight-channel sodium array by managing their respective resonances and geometrical arrangement.

The eight-channel coil was constructed to cover the knee cartilage $(15 \mathrm{~cm}$ length) on $0.8 \mathrm{~mm}$ thick FR4 copper-clad circuit board with $0.8 \mathrm{~cm}$ conductor width and arranged on a close-fitting cylindrical acrylic former with $20.3 \mathrm{~cm}$ diameter, as depicted in Figure 11.

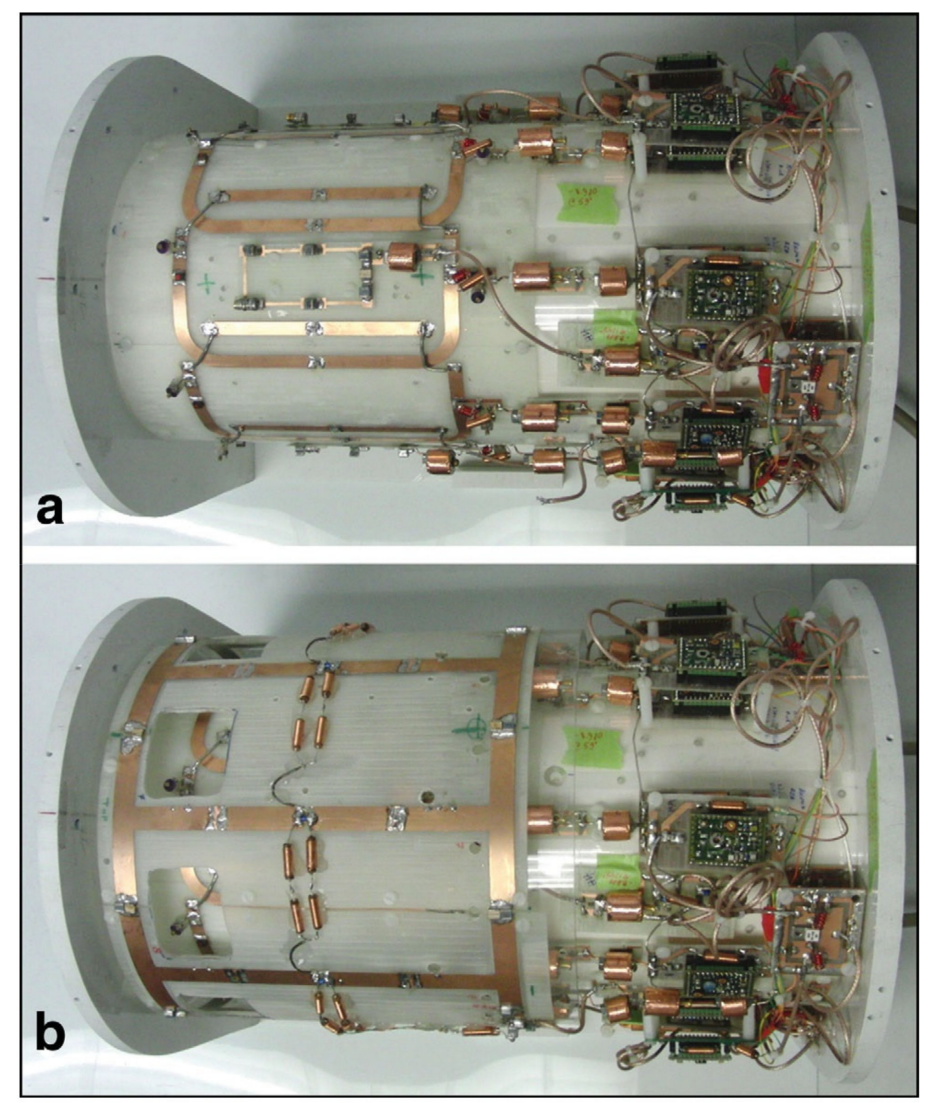

Figure 11. (a)The eight-channel sodium receive array and four-channel transmit-receive hydrogen array on the inner former; (b) the detunable sodium birdcage on the outer former. Reprinted by permission from Brown et al., Magn Reson Med 2013 [32]. 
Each coil was tuned to the sodium resonance frequency at $7 \mathrm{~T}(78.6 \mathrm{MHz})$ using four distributed capacitors and matched to $50 \mathrm{Ohm}$ while loaded with a cylindrical phantom. A PIN diode circuit was used to actively detune the sodium transmit coils and sodium transmit and receive coil. The output of each coil was connected to a low-impedance preamplifier using coaxial cable of appropriate length for preamplifier decoupling.

Graessl [33] proposed a dual-frequency RF coil setup consisting of separate elements for sodium and hydrogen MR for a $7 \mathrm{~T}$ whole body MR scanner. Hydrogen/sodium RF coil array tailored for cardiac sodium $\mathrm{MR}$ at $7 \mathrm{~T}$ with four channels for sodium was constructed to conform to an anterior and superior chest section. Each section contains two rectangular loop elements $\left(210 \times 140 \mathrm{~mm}^{2}\right)$, etched from $32 \mu \mathrm{m}$ copper on $1 \mathrm{~mm}$ FR4 substrate. The adjustable decoupling of the neighboring loop elements was obtained with a trimmer capacitor integrated in the shared conductor. Cable traps for hydrogen were designed as double-turn solenoids of the coaxial cable with an appropriate capacitor soldered to the outer conductor at the crossing of the cable.

Basic hydrogen imaging capabilities were achieved by a dipole bow tie element tuned to $297 \mathrm{MHz}$ placed inside the anterior section of the sodium coil.

Measurements and characterization of the RF coil performance were performed using an eight-channel vector network analyzer. A high value of quality factor ratio $r$ of the loop elements indicated a predominant impact of sample noise over coil noise.

The eight-channel dual tuned sodium / hydrogen coil design for human torso [34] and abdomen [35] imaging on $3 \mathrm{~T}$ consisted of two identical top and bottom plates $\left(30 \times 30 \mathrm{~cm}^{2}\right)$. Each plate consisted of one sodium Tx loop (that provides a relatively homogenous RF excitation and deep penetration) and four sodium Rx elements (that provided a high fillingfactor and SNR). Each coil plate also consisted of a hydrogen Tx/Rx loop arranged around the sodium Rx arrays for hydrogen for co-registration imaging (Figure 12). The hydrogen and the sodium transmit coils on the anterior and posterior plates formed Helmholtz pairs and shared one transmit connector which helped in distributing the power to the two coils using this single connector. The transmit signal passed through a frequency filter and splitter to the respective hydrogen and sodium transmit circuits.
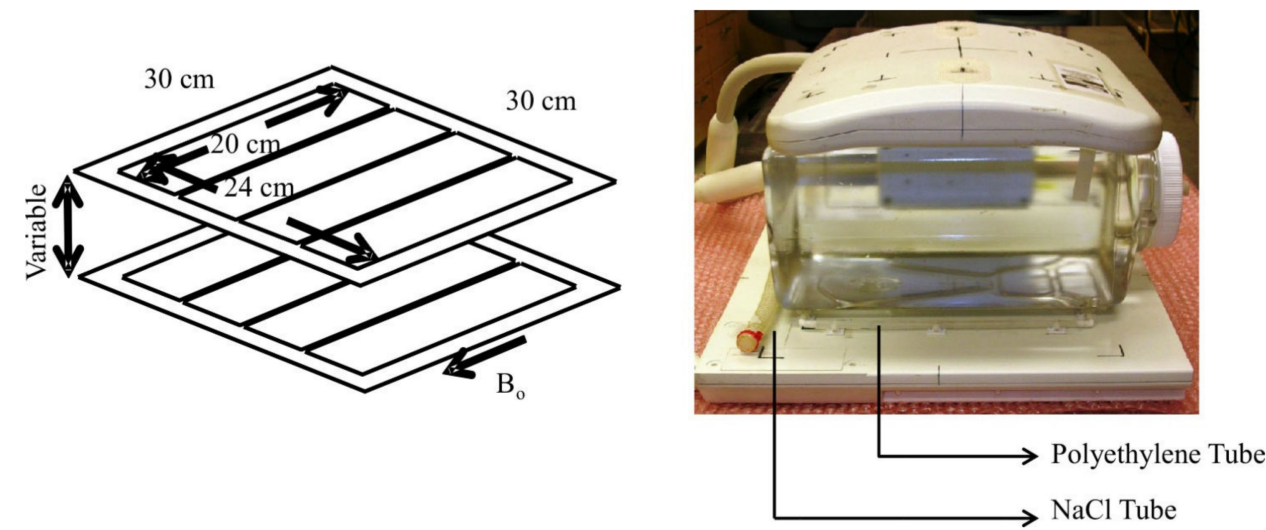

Figure 12. The eight-channel dual-tuned sodium and hydrogen torso coil with the polyethylene and $\mathrm{NaCl}$ tubes employed for co-registration and signal intensity reference for estimating tissue sodium concentration. Reprinted by permission from James et al., Abdom Imag 2015 [35].

Specific Absorption Rate (SAR) for the eight-channel dual tuned sodium/hydrogen coil at both sodium and hydrogen frequencies was measured using the FDA recognized 'Calorimetric Method' with a phantom of $50 \mathrm{mM} \mathrm{NaCl}$ and $0.5 \mathrm{mM}$ Omniscan Gadodiamide, and showed that the coil produced a temperature increase of $1.1^{\circ} \mathrm{C}$ during sodium MRI and $1.0^{\circ} \mathrm{C}$ during hydrogen MRI over 1 hour of acquisition. These temperature changes correspond to the SAR of $1.1 \mathrm{~W} / \mathrm{kg}$ at sodium frequency and $1.2 \mathrm{~W} / \mathrm{kg}$ at hydrogen frequency in comparison to the maximum safe SAR of $4 \mathrm{~W} / \mathrm{kg}$ recommended by FDA for torso and head MRI. 
The eight-channel dual-tuned sodium/hydrogen coil gave approximately two times better average SNR than the in-built body coil and provided better shimming compared to the body coil with both automatic 3D phase mapping technique and manual adjustments of shim currents.

In vivo 3D sodium and hydrogen images of healthy volunteers were acquired: the sodium images had reasonably good resolution and SNR considering that the sodium signal in tissue is $\sim 10$ times weaker than the water hydrogen signal. 3D. Furthermore, it was possible to acquire sodium images of the human torso with the eight-channel sodium/hydrogen coil without moving patients.

Kim [36] applied a multi transceiver RF coil design to develop a dual tuned hydrogen/sodium coil for knee cartilage imaging at $3 \mathrm{~T}$, to take advantage of both surface and volume coil effects and to offer high SNR and homogeneity.

The coil structure consisted of four hydrogen and eight sodium loops (greater than the hydrogen channels to enhance and improve sodium signal). Eight sodium coil loops (rectangle $135 \times 85 \mathrm{~mm}^{2}$ ) were constructed at the inner cylindrical plastic tube, and four hydrogen coil loops (rectangle $180 \times 130 \mathrm{~mm}^{2}$ ) were attached to the outer cylindrical tube. The reflection coefficient $\left(\mathrm{S}_{11}\right)$ and transmission coefficient $\left(\mathrm{S}_{12}\right)$ were estimated in hydrogen-only $\left(\mathrm{S}_{11}=-22 \mathrm{~dB}\right.$ and $\mathrm{S}_{11}=-20 \mathrm{~dB} ; \mathrm{S}_{21} \sim 17$ for both).

In the sodium imaging, the mean $\mathrm{S} 11$ was $-22.3 \mathrm{~dB}$ for the sodium-only coil and $-21.1 \mathrm{~dB}$ for the sodium DT coil, while the mean S12 was $-20.4 \mathrm{~dB}$ for the sodium-only coil and $-19.5 \mathrm{~dB}$ for the sodium DT coil. The $\mathrm{B}_{1}$ magnetic field distribution Tx and apparent Rx fields were measured from the homogeneous phantom imaging. In the sodium imaging, the Tx field was more homogeneous than the Rx field, although the difference between the Tx and apparent Rx fields (the inhomogeneity index Tx $11 \%$ vs. Rx 30\%) was greater than that of the hydrogen imaging (i.e., the inhomogeneity index Tx 15\% vs. Rx 18\%).

For in vivo sodium MR imaging of the knee, the Tx field distribution was measured using the same ROI as the phantom study. The measured mean Tx field inhomogeneity was $11.8 \%$ for the dual tuned coil.

The sodium imaging SNR with the DT coil increased symmetrically from the center to the periphery (regional mean SNRs ranged $23-55$ with the mean 36 ) and for in vivo imaging the mean SNRs of cartilages ranged from 27 to 38 . The mean Contrast-to-Noise Ratio (CNR) of cartilages to bone were 15 to 21 using DT coil.

Knee hydrogen images with water excitation, cartilages and menisci were well delineated from the surrounding tissues. High sodium signal intensity of cartilages was conspicuously demarcated on the co-registered sodium/hydrogen images.

Lakshmanan [37] built an array of eight triangular loops with a height of $17.8 \mathrm{~cm}$ $(\mathrm{H} / \mathrm{F})$ and base of $17.8 \mathrm{~cm}$ on the elliptical cylinder which fitted closely to the human head to maximize loading, with $r$ ratio of $\sim 2.5$. They implemented triangular elements that provided the means to decouple next-nearest-neighbor coils using counter-wound inductors at the shared triangular vertices (Figure 13).

The triangular array was connected to the scanner by using a one-to-eight-way power splitter. In order to obtain circularly polarized transmit excitation, home-built lumped element phase shift circuits were added for providing $45^{\circ}$ phase offsets as according to the coil elements azimuthal location. The decoupling of preamplifier was carried out with the insertion of a phase shift network in the receive path which transformed the low input impedance of the preamplifier into an inductance which formed a parallel resonant circuit with the matched capacitor. A sketch of the eight-channel sodium/hydrogen coil array and interface is shown in Figure 14. 


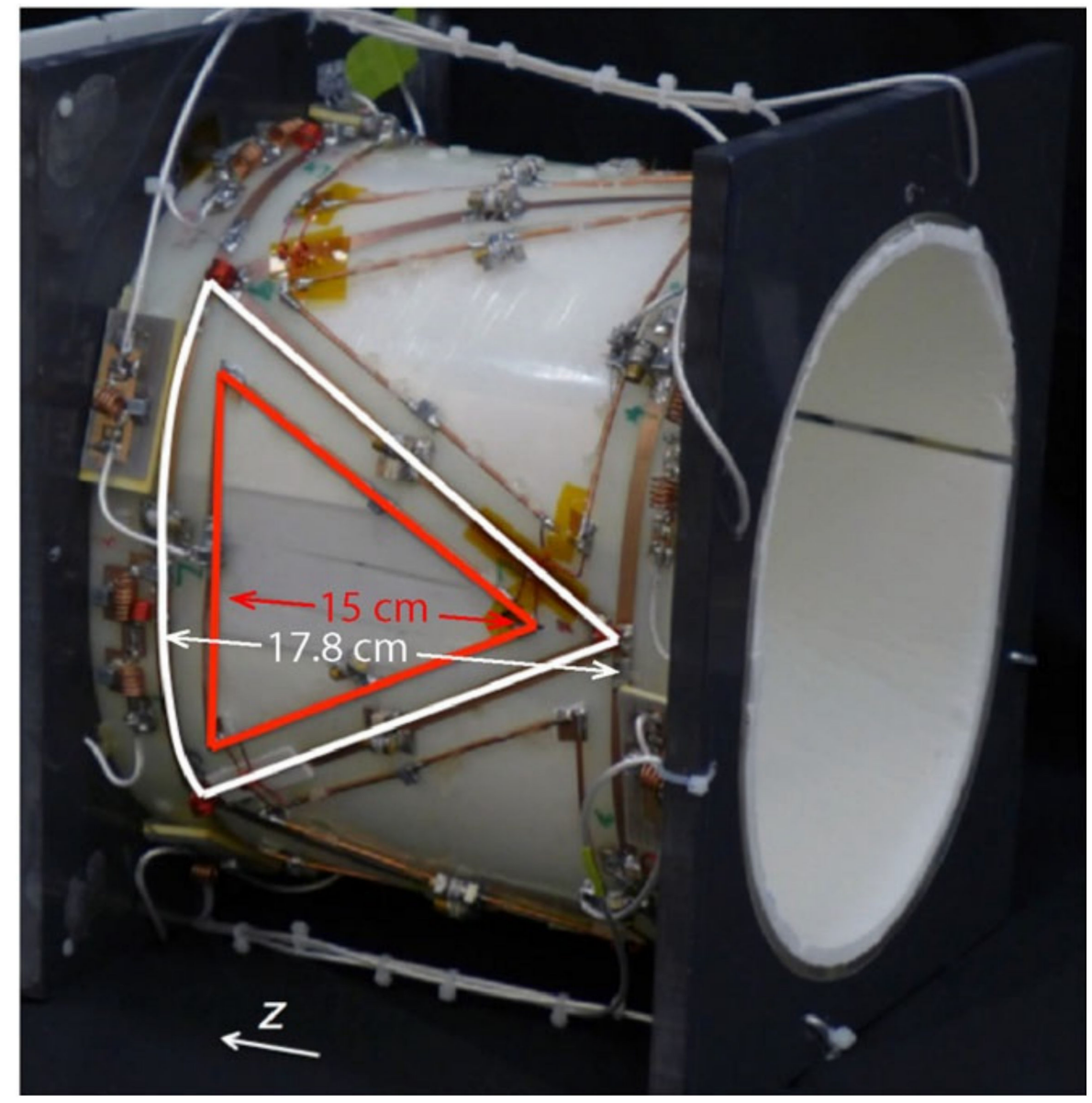

Figure 13. The eight-channel sodium/hydrogen coil array, where one sodium and one hydrogen element are coloured in white and red, respectively. Reprinted by permission from Lakshmanan et al., NMR in Biomed 2017 [37].

Finite difference time domain method was employed for comparing the efficiency of the triangular geometry $(17.8 \mathrm{~cm}$ in height and $17.8 \mathrm{~cm}$ in base) with that provided by a rectangular loop $(8.9 \mathrm{~cm} \mathrm{L/R} \times 17.8 \mathrm{~cm} \mathrm{H} / \mathrm{F})$.

Results showed that the reflection coefficient for one element of a three-channel rectangular array contained a split resonance typical of strong coupling between nextnearest-neighbor coils, while the decoupling inductors in a triangular array remove such coupling, by resulting in a single resonance. They simulated also the sodium $\mathrm{B}_{1+}$ fields to demonstrate that the triangular loop efficiency was about $90 \%$ of the rectangular loop at a depth of $150 \mathrm{~mm}$ along the main axis, probably due to a higher $\mathrm{z}$-directed magnetic fields generated by the angled rungs of the triangular respect to rectangular element. 


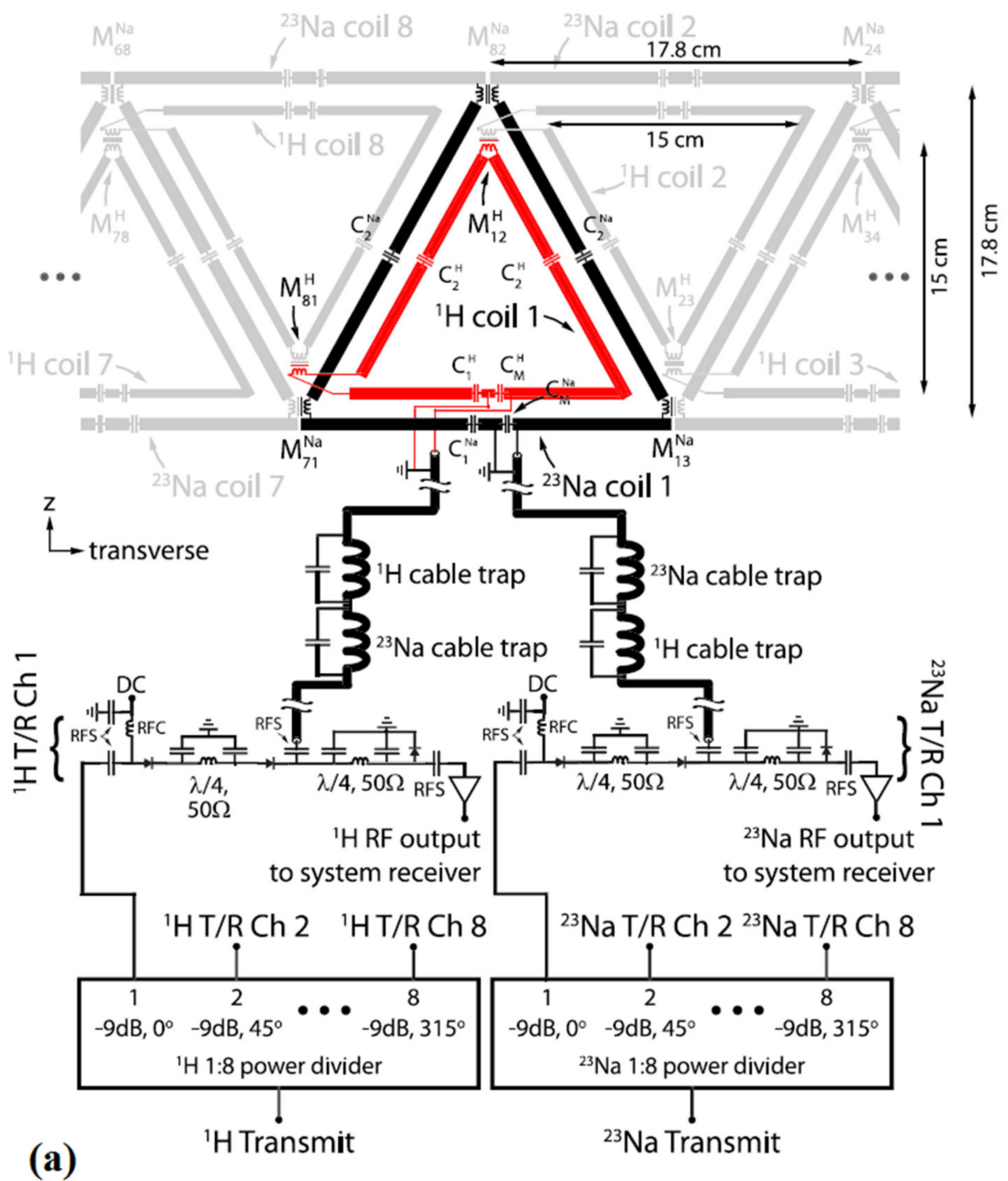

(a)

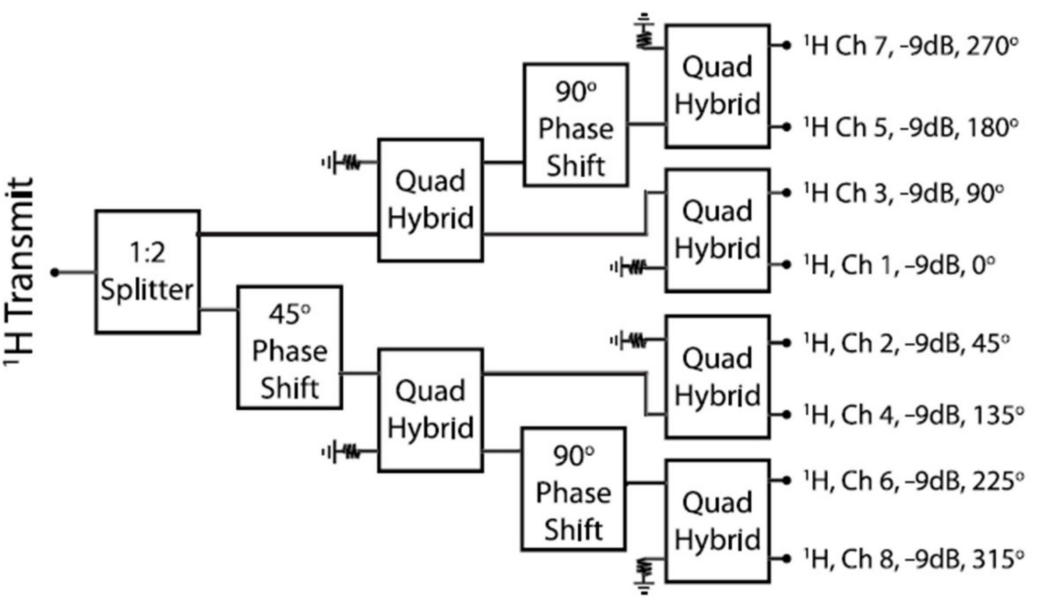

(b)

Figure 14. (a) Flattened two-dimensional schematic diagram of the eight-channel sodium/hydrogen coil array and interface, in which a single sodium (black) and hydrogen coil (red) are highlighted; (b) block diagram of the hydrogen power divider. Reprinted by permission from Lakshmanan et al., NMR in Biomed 2017 [37]. 
Malzacher [38] aimed the paper at the description of a double resonant receive ( $\mathrm{Rx})$ coil array for hydrogen and sodium head MRI. The sodium coil, showed in Figure 15, is a degenerate birdcage coil with eight independent Rx channels (8Rx sodium and 8Rx hydrogen).

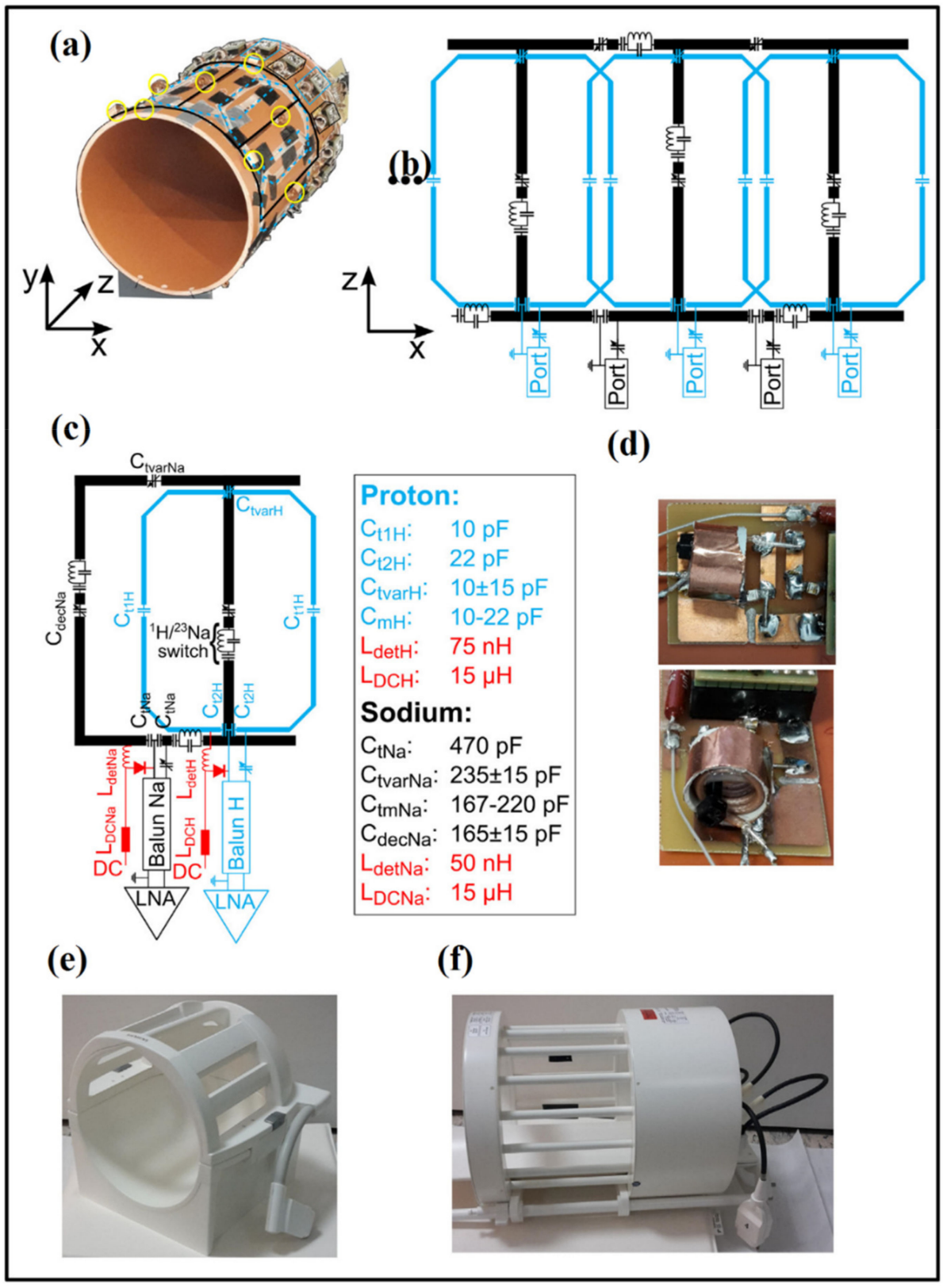

Figure 15. (a) Image of the $8 \mathrm{Rx}$ hydrogen/8Rx sodium coil, where the sodium coil is highlighted with the solid black lines and the hydrogen coil with the dotted blue lines; (b) part of the coil circuit with two sodium elements (in black) and three hydrogen elements (in blue); (c) sketch of a single sodium (black) and a single hydrogen (blue) coil element; (d) cable trap in a top and a front view; (e) Image of the commercial 12Rx coil; (f) image of the commercial TxRx hydrogen/sodium coil. Reprinted by permission from Malzacher et al., Magn Reson Imag 2019 [38].

The coil structure was a PVC tube (outer diameter $=250 \mathrm{~mm}$ ) on which was mounted a flat copper conductor (width $=6 \mathrm{~mm}$, thickness $=0.1 \mathrm{~mm}$ ). The coil elements were split by capacitors two in the ring segments (used to tune the individual coil) and two in the legs (used to decouple nearest neighboring coil).

A sodium cable trap (made with coaxial cable bridged by a capacitor and shielded with copper tape) was positioned between the coil element and the preamplifier. 
Each coil element was tuned and matched and preamplifier decoupling was adjusted by varying the series and parallel capacitor of the matching circuit iteratively.

The hydrogen $\mathrm{Rx}$ array (8Rx coil) were $131 \mathrm{~mm}$ in x-direction and $216 \mathrm{~mm}$ in $\mathrm{z}$ direction and each coil element was built in an octagonal shape to decrease capacitive coupling at the overlap positions. Moreover, coil elements were smaller than the $8 \mathrm{Rx}$ sodium coil elements to decrease coupling between the two nuclei. The optimal overlap was found changing gradually until a decoupling of at least $-15 \mathrm{~dB}$ was reached.

Each coil element was connected to the preamplifier via a capacitive matching network.

Between the coil element and the preamplifier, a hydrogen cable trap was placed. The overlap distance was checked for optimum decoupling using transmission S-parameter measurements.

The transmission parameter achieved for sodium was $-0.8 \mathrm{~dB}$ and $-62.4 \mathrm{~dB}$ at the hydrogen frequency. The reflection parameters at the sodium frequency measured were $-42.6 \mathrm{~dB}$ and $-1.7 \mathrm{~dB}$ at the hydrogen frequency.

Coupling between the hydrogen and sodium coil elements was in the worst case $-60 \mathrm{~dB}$ at the hydrogen frequency $(123.2 \mathrm{MHz})$ and $-50 \mathrm{~dB}$ at the sodium frequency $(32.6 \mathrm{MHz})$.

The decoupling between nearest coil elements of the sodium array was $-16.4 \pm 2.4 \mathrm{~dB}$, while between nearest coil elements hydrogen array was $-16 \pm 3.4 \mathrm{~dB}$ (worst case $-12.5 \mathrm{~dB})$.

The performance of the $8 \mathrm{Rx}$ hydrogen/ $8 \mathrm{Rx}$ sodium coil was evaluated with phantom and in-vivo measurements (optimized for head studies).

In phantom and in vivo brain acquisitions, the SNR of the $8 R x$ hydrogen/8Rx sodium coil resulted comparable to the one provided by the commercial coil (TxRx hydrogen/sodium).

The sodium SNR performance was slightly better than the SNR performance of the commercial coil despite some SNR decrease in the neck region. This combination of high sodium sensitivity and full hydrogen imaging capability makes an important contribution towards clinically used sodium MRI due to a more optimized and faster workflow.

\section{Pulse Sequences and Image Reconstruction}

The sodium nucleus has a two-third spin, and typically exhibits rapid, bi-exponential MR signal decay in biological tissues [39]. This needs pulse sequences with very short echo time (TE) and places significant limits on signal readout duration, leading to image blurring and loss of SNR.

Sodium tissue MR signals can have multiple contributing T2 components. It is unclear to what extent the scatter in these values reflect real physiological variability, or just the difficulty and error in measuring them by sodium MR [14].

Certainly, the use of ultra-short sodium MRI TEs would avoid the uncertainty in T2 and minimize the SNR loss from T2 decay. Ultra-short TE sodium MRI pulse sequences and their associated reconstruction algorithms generally require customized programming that makes the application difficult in clinical routine.

The simplest ultra-short TE approach used for detecting and imaging sodium is threedimensional (3D) radial acquisition [40]. This sequence comprises a short, hard excitation pulse, followed by a constant radial gradient that is reoriented in subsequent applications, to generate a 3D set of angular projections. By eliminating the slice-selection and phaseencoding gradients with their associated delays, this pulse sequence allows to achieve ultra-short TEs $(0.2-0.4 \mathrm{~ms})$. Additionally, the constant gradient radial projections do over-sample the central low-spatial resolution Fourier components, and under-sample the high spatial frequency components [41]. The 3D radial is not the most efficient trajectory for covering image k-space. Spiral and "twisted projection imaging", or TPI, are nonCartesian acquisition schemes that have been used in particular for human cardiac sodium MRI [42,43].

One significant problem with all sequences using hard pulses, is the effect of RF field $\left(B_{1}\right)$ inhomogeneity. This may arise, for example, when using surface coils for exciting 
the sodium MR signal. The resultant spatially varying excitation flip-angle (FA) could be addressed by calibrating the spatial $\mathrm{B}_{1}$ distribution, or by replacing the hard pulse with an adiabatic pulse to provide substantially $B_{1}$-independent excitation [44].

Additional challenges of in-vivo cardiac sodium imaging are cardiac and respiratory motion as well as partial volume (PV) effects caused by the low spatial resolution and large differences in sodium concentration between blood compartments and myocardial tissue. To address these issues, several correction methods have been proposed to get accurate quantification of tissue sodium concentration in the heart [45].

In general, the inherent low sensitivity of sodium requires long acquisition times to obtain adequate SNR and resolution. Scan time reduction can be achieved using the compressed sensing (CS) technique, i.e., the iterative reconstruction from highly undersampled data based on sparsity and incoherence, i.e., noise-like, undersampling artifacts. Recently, wavelet-based CS was shown to enable fourfold acceleration in sodium imaging, while maintaining high image quality and structural detail [46].

Sodium MRI signal exhibit a complex multi-exponential T2 * relaxation due to its quadrupole moments.

A detailed description of this phenomenon goes beyond the scope of this work; hence we refer the interested reader to the literature [47-49]. Here we are just interested in the possible shapes of the relaxation and in how this affects the quantification of the sodium signal.

If sodium nuclei, are free to move (the motional correlation time is lower than Larmor periods) they exhibit a mono-exponential $\mathrm{T} 2 *$ decay instead if hindered in movement (the motional correlation time is higher than Larmor periods) they exhibit a bi-exponential decay. In real samples, due to tissue heterogeneity, different sodium pools could exist in neighbor voxels or even coexist in the same voxel; one of those heterogonous voxels could have more than two exponential decay components.

Blunck [50] proposed a two-step method. Initially, all the voxels were fit with a biexponential model with fixed components ratio. Then they check if the faster decay $\mathrm{T} 2 *$ is higher than a tissue depending threshold. Finally, they fit all the voxel whose faster decay exceeds the threshold with a mono-exponential model. While theoretically correct for an idealized sample this model could be too rigid to handle heterogeneous tissue, e.g., when there is more than one sodium pool inside the voxel due to partial volume effect.

Riemer [51] proposed to solve the Inverse Laplace Transform (ILT) problem to directly study the distribution of relaxation time using a CONTIN-like method. This model is flexible and could adapt to highly heterogeneous tissue. Unfortunately, the ILT problem is a well-known ill-posed problem therefore, the solution is strongly dependent on the quality of the experimental data, on the algorithm used to solve the ILT problem, and on the previous knowledge on the system used to set up the inversion problem.

Finally, Syeda [52] proposed to model the T2 * population distribution with a Gamma distribution, whose Laplace transform is fitted to the experimental data. The authors selected the Gamma distribution for its flexible shape and due to the existence of an analytical solution for its Laplace Transform. This model lacks a theoretical justification but is more robust than solving the general ILT problems.

\section{Clinical Applications of Sodium MR}

In a review on sodium magnetic resonance imaging, Thulborn K.R. [53] wrote the following sentence: "sodium MR imaging can significantly extend clinical MR imaging beyond anatomic changes to earlier stages of disease and to diseases without an anatomic basis". This sentence highlights the fact that we should have to look at sodium MR not as an imaging technique defining anatomical burden of disease, but we should have to look beyond this through the development of new parameters of cell viability and metabolism based on the cellular dynamic of sodium. This electrolyte is a cornerstone of cell viability and metabolism. Tissue sodium concentration (TSC) can be calculated both at 
the intracellular and extracellular compartments, and this can allow to assess the extent and quality of extracellular matrix as well as cell volume fraction.

Clinical perspectives of sodium MRI can be reassumed in the following points:

- The tissue viability assessment. Sodium provides a marker of tissue viability in terms of the TSC that can be considered a direct biochemical marker of cellular function and viability. Thus, TSC could be considered a new parameter of tissue viability with the potential to provide additive information regarding the entity and severity of damaged tissue as well as the probability of recovery. In the brain a TSC increases at least $50 \%$ in the infarcted area in comparison to non-infarcted one. This means that TSC is around $70 \mathrm{mmol} / \mathrm{L}$ in comparison the concentration of around $45 \mathrm{mmol} / \mathrm{L}$ in normal tissue [53]. The TSC increase in continuous in the early phases of stroke and this can reduce the efficacy of reperfusion [4]. Interestingly, in an animal study of acute stroke, a TSC less than $55 \mathrm{mmol} / \mathrm{L}$ was associated a higher probability of recovery [53]. Regarding skeletal muscle, there are evidences showing that TSC can be assessed in patients with diseased muscle. Interestingly, TSC accumulation has been also observed in subjects after exercise [54]. This may be due to a physiological inhibition of the $\mathrm{Na} / \mathrm{K}$ adenosine triphosphatase as a consequence of energy exhaustion, or to a change in sodium interactions with other molecules accumulating in the skeletal muscles after exercise [55].

- The increase in TSC has been also observed in an animal model of myocardial infarction. The peak of this increase occurs within the first day after induced myocardial infarction and reduces progressively in the following days due to the healing process [56]. Similarly, an increase in myocardial TSC has been documented in patients with chronic myocardial infarction $[57,58]$. There was no correlation between myocardial TSC and infarct size, left ventricular function and also with the occurrence of ventricular arrhythmia [59]. However, these are initial results shown in a small patient population and, thus, need to be confirmed.

- The applications in therapy. The sodium has the potential to assess the effectiveness of the therapeutical approaches to minimize or reverse organ's lesion. The increase in TSC has been also documented in tumors. This is due to cell proliferation and also to the higher extent of extracellular matrix. In the brain tumors, extracellular matrix is increased whereas cell volume fraction is reduced in comparison to normal brain tissue. The effectiveness of the different cycles of oncogenic therapy could be assessed through the measurement of the cell volume fraction [58].

- The role of sodium MRI in the prevention policies. Initial studies were performed on the role of sodium content in skin and skeletal muscle in hypertension. In this context, TSC measurement could provide new information on the relationship between sodium and dietary salt consumption and cardiovascular risk, as well as the effects of antihypertensive treatment. Kopp et al. [60] measured TSC in skin and skeletal muscle in subjects with primary aldosteronism and found a $29 \%$ increase in muscle sodium content whereas skin Na sodium was basically but not significantly higher. Interestingly TSC in the muscle decreased after spironolactone treatment. In another study, Kopp C. et al. [61] measured water and sodium content both in skin and muscle showing water free sodium storage in muscle, and a paralleled increase in water and sodium in skin. Interestingly, sodium content increased with age in skin and muscle both in women and men, but skin increase was less pronounced in women. Moreover, in the same study, the authors showed that refractory hypertension was associated with sodium accumulation that reduced with spironolactone treatment. Moreover, myocardial TSC accumulation has also been recently documented in patients with primary hyperaldosteronism [62]. This accumulation reduced significantly after treatment. Tissue sodium accumulation has been documented also in patients with diabetes [63]. Recently, sodium glucose cotransporter 2 (SGLT-2) inhibitors, a new antidiabetic drug, that decreases reabsorption of glucose in the renal tubular system, parallel blocking sodium reabsorption, reduced sodium skin content in dia- 
betic patients [64]. A recent study showed high reliability and agreement of sodium content in skin and skeletal muscle in healthy subjects, a key result to apply this new technique in the clinical practice [65].

Furthermore, a potential sodium MR application is the detection of nerve activity directly through the assessment of neuronal electric currents. Currently functional MRI (fMRI) detects nerve activity indirectly through the evaluation of hemodynamic changes, i.e., blood flow, or blood volume or blood oxygenation, occurring in the activated brain area with the Blood Oxygen Level Dependent (BOLD) method. Sekino SY et al. [66] showed an increase in the concentration of extracellular sodium/intracellular sodium ions in the sciatic nerves from bull frogs during electrical nerve stimulation as a direct change in sodium ions permeability.

In general, sodium tissue quantification represents a challenge to increase our knowledge on physiological and pathophysiological processes in which sodium is involved. This may facilitate assessment of diagnostic and prognostic stratification in patients with several acute and chronic organ diseases, therapeutical decisions, and also environmental factors, i.e., diet exercise, for primary and secondary prevention

\section{Conclusions}

Compared to hydrogen MRI, sodium is the second most viable nucleus for performing routine human MRI in its natural endogenous form. The detection of sodium signals in vivo is challenging; indeed, sodium images typically have low resolution, low SNR and long acquisition times, limited by the hardware capabilities of clinical scanners (currently around $40 \mathrm{mT} / \mathrm{m})$ and physiological constraints on gradient slew rates $(200 \mathrm{~T} /(\mathrm{ms}))$. Such constraints in sodium imaging require technical improvements as strong magnetic field gradients, pulse sequence programming and image reconstruction. Furthermore, when setting up the scanner to work in a different frequency range it is necessary to consider the use of dedicated RF coils.

In this paper we reviewed the theoretical basis of sodium coil including simulation and workbench test methods and the different coil typologies (surface, volume and phasedarray coils) described in literature for different in vivo applications, by providing details of the design, modeling and construction of such coils.

A separate section was dedicated to the different pulse sequences and image reconstruction methods for in vivo application, underlines that provide additional sensitivity and contrast enhancements, while final considerations were also given regarding the clinical applications of sodium MR.

There are definite advantages to sodium MRI, and from the many promising studies that have been performed we can expect many new applications with of sodium MRI to emerge in the future; for this reason we believe that this review could be of interest to the readers working with sodium Magnetic Resonance Imaging and Spectroscopy, since the provided information permits them to choose the coil geometry for particular needs.

Author Contributions: Coil design and development, conception of the review paper, collecting the data and drafting of the manuscript, G.G. and F.F.; image analysis of MRI data and contribution to write the manuscript, A.F., N.M. and R.F.; clinical applications and contribution to write the manuscript, G.D.A. and A.P. All authors have read and agreed to the published version of the manuscript.

Funding: This research received no external funding.

Conflicts of Interest: The authors declare no conflict of interest. 


\section{References}

1. Thulborn, K.R. Quantitative Sodium MR Imaging: A Review of its Evolving Role in Medicine. Neuroimage 2018, 168, 250-268. [CrossRef] [PubMed]

2. $\quad$ Ouwerkerk, R.; Jacobs, M.A.; Macura, K.J.; Wolff, A.C.; Stearns, V.; Mezban, S.D.; Khouri, N.F.; Bluemke, D.A.; Bottomley, P.A. Elevated tissue sodium concentration in malignant breast lesions detected with non-invasive 23Na MRI. Breast Cancer Res. Treat. 2007, 106, 151-160. [CrossRef] [PubMed]

3. Poku, L.O.; Phil, M.; Cheng, Y.; Wang, K.; Sun, X. 23Na-MRI as a Noninvasive Biomarker for Cancer Diagnosis and Prognosis. J. Magn. Reson. Imaging 2021, 53, 995-1014. [CrossRef]

4. Hussain, M.S.; Stobbe, R.W.; Bhagat, Y.A.; Emery, D.; Butcher, K.S.; Manawadu, D.; Rizvi, N.; Maheshwari, P.; Scozzafava, J.; Shuaib, A.; et al. Sodium imaging intensity increases with time after human ischemic stroke. Ann. Neurol. $2009,66,55-62$. [CrossRef]

5. Boada, F.E.; Qian, Y.; Nemoto, E.; Jovin, T.; Jungreis, C.; Jones, S.C.; Weimer, J.; Lee, V. Sodium MRI and the Assessment of Irreversible Tissue Damage During Hyper-Acute Stroke. Transl. Stroke Res. 2012, 3, 236-245. [CrossRef]

6. Petracca, M.; Fleysher, L.; Oesingmann, N.; Inglese, M. Sodium MRI of multiple sclerosis. NMR Biomed. 2016, $29,153-161$. [CrossRef] [PubMed]

7. Weber, C.E.; Nagel, K.; Ebert, A.; Roßmanith, C.; Paschke, N.; Adlung, A.; Platten, M.; Schad, L.R.; Gass, A.; Eisele, P. Diffusely appearing white matter in multiple sclerosis: Insights from sodium (23Na) MRI. Mult. Scler. Relat. Disord. $2021,49,102752$. [CrossRef]

8. Mohamed, S.A.; Herrmann, K.; Adlung, A.; Paschke, N.; Hausner, L.; Frölich, L.; Schad, L.; Groden, C.; Kerl, H.U. Evaluation of Sodium (23Na) MR-imaging as a Biomarker and Predictor for Neurodegenerative Changes in Patients With Alzheimer's Disease. In Vivo 2021, 35, 429-435. [CrossRef] [PubMed]

9. Madelin, G.; Regatte, R.R. Biomedical Applications of Sodium MRI In Vivo. J. Magn. Reson. Imaging 2013, 38, 511-529. [CrossRef] [PubMed]

10. Zaric, O.; Juras, V.; Szomolanyi, P.; Schreiner, M.; Raudner, M.; Giraudo, C.; Trattnig, S. Frontiers of Sodium MRI Revisited: From Cartilage to Brain Imaging. J. Magn. Reson. Imaging 2021, 54, 58-75. [CrossRef]

11. Madelin, G.; Kline, R.; Walvick, R.; Regatte, R.R. A method for estimating intracellular sodium concentration and extracellular volume fraction in brain in vivo using sodium magnetic resonance imaging. Sci. Rep. 2014, 4, 4763. [CrossRef] [PubMed]

12. Fleysher, L.; Oesingmann, N.; Brown, R.; Sodickson, D.K.; Wiggins, G.C.; Inglese, M. Non-invasive Quantification of Intracellular Sodium in Human Brain using Ultra-High-Field MRI. NMR Biomed. 2013, 26, 9-19. [CrossRef]

13. Ianniello, C.; Moy, L.; Fogarty, J.; Schnabel, F.; Adams, S.; Axelrod, D.; Axel, L.; Brown, R.; Madelin, G. Multinuclear MRI to disentangle intracellular sodium concentration and extracellular volume fraction in breast cancer. Sci. Rep. 2021, 11, 5156. [CrossRef]

14. Bottomley, P.A. Sodium MRI in human heart: A review. NMR Biomed. 2016, 29, 187-196. [CrossRef]

15. Wiggins, G.C.; Brown, R.; Lakshmanan, K. High-performance radiofrequency coils for 23Na MRI: Brain and musculoskeletal applications. NMR Biomed. 2016, 29, 96-106. [CrossRef] [PubMed]

16. Jin, J. Electromagnetic Analysis and Design in Magnetic Resonance Imaging; Routledge \& CRC Press: Abingdon, UK, 1999.

17. Vaughan, J.T.; Griffiths, J.R. RF Coils for MRI; Wiley: Hoboken, NJ, USA, 2012.

18. Roemer, P.B.; Edelstein, W.A.; Hayes, C.E.; Souza, S.P.; Mueller, O.M. The NMR phased array. Magn. Reson. Med. 1990, 16, 192-225. [CrossRef] [PubMed]

19. Gruber, B.; Froeling, M.; Leiner, T.; Klomp, D.W.J. RF coils: A practical guide for nonphysicist. J. Magn. Reson. Imaging 2018, 48, 590-604. [CrossRef]

20. Chen, C.N.; Hoult, D.I. Biomedical Magnetic Resonance Technology; Adam Hilger: Bristol, UK, 1989.

21. Giovannetti, G.; Viti, V.; Positano, V.; Santarelli, M.F.; Landini, L.; Benassi, A. Magnetostatic simulation for accurate design of low field MRI phased-array coils. Concepts Magn. Reson. Part. B Magn. Reson. Eng. 2007, 31, 140-146. [CrossRef]

22. Giovannetti, G.; Hartwig, V.; Positano, V.; Vanello, N. Radiofrequency coils for magnetic resonance applications: Theory, design, and evaluation. Crit. Rev. Biomed. Eng. 2014, 42, 109-135. [CrossRef]

23. Anisimov, N.V.; Sadykhov, E.G.; Pavlova, O.S.; Fomina, D.V.; Tarasova, A.A.; Pirogov, Y.A. Whole Body Sodium MRI at 0.5 Tesla Using Surface Coil and Long Echo Time Sequence. Appl. Magn. Reson. 2019, 50, 1149-1161. [CrossRef]

24. Giovannetti, G.; Pingitore, A.; Positano, V.; De Marchi, D.; Valvano, G.; Gibiino, F.; Aquaro, G.D.; Lombardi, M.; Landini, L.; Santarelli, M.F. Improving sodium Magnetic Resonance in humans by design of a dedicated ${ }^{23} \mathrm{Na}$ surface coil. Measurement 2014, 50, 285-292. [CrossRef]

25. Maril, N.; Rosen, Y.; Reynolds, G.H.; Ivanishev, A.; Ngo, L.; Lenkinski, R.E. Sodium MRI of the Human Kidney at 3 Tesla. Magn. Reson. Med. 2006, 56, 1229-1234. [CrossRef]

26. Maggiorelli, F.; Buonincontri, G.; Retico, A.; Kaggie, J.D.; Graves, M.J.; Biagi, L.; Tiberi, G.; Tosetti, M. Sodium imaging of the human knee cartilage with Magnetic Resonance at Ultra High Field: Development of a double frequency $(1 \mathrm{H} / 23 \mathrm{Na}) \mathrm{RF}$ coil. In Proceedings of the International Applied Computational Electromagnetics Society Symposium Italy (ACES) 2017, Firenze, Italy, 26-30 March 2017; pp. 1-2. 
27. Wetterling, F.; Corteville, D.M.; Kalayciyan, R.; Rennings, A.; Konstandin, S.; Nagel, A.M.; Stark, H.; Schad, L.R. Whole body sodium MRI at 3T using an asymmetric birdcage resonator and short echo time sequence: First images of a male volunteer. Phys. Med. Biol. 2012, 57, 4555-4567. [CrossRef]

28. Wetterling, F.; Rennings, A.; Kalayciyan, R.; Corteville, D.M.; Konstandin, S.; Schad, L.R. A dual resonator system for whole-body sodium-MRI at 3T. Proc. Intl. Soc. Mag. Reson. Med. 2011, 19, 471.

29. Giovannetti, G.; Valvano, G.; Virgili, G.; Giannoni, M.; Flori, A.; Frijia, F.; De Marchi, D.; Hartwig, V.; Landini, L.; Aquaro, G.D.; et al. Design and simulation of a dual-tuned $1 \mathrm{H} / 23 \mathrm{Na}$ birdcage coil for MRS studies in human calf. Appl. Magn. Reson. 2015, 46, 1221-1238. [CrossRef]

30. Qian, Y.; Zhao, T.; Wiggins, G.C.; Wald, L.L.; Zheng, H.; Weimer, J.; Boada, F.E. Sodium Imaging of Human Brain at 7T with 15-Channel Array Coil. Magn. Reson. Med. 2012, 68, 1808-1814. [CrossRef]

31. Robson, M.D.; Titus, L.; Neubauer, S. Cardiac sodium imaging with phased arrays at 3 Tesla using a 3D Ultra-short TE (UTE) approach. J. Cardiov. Magn. Reson. 2008, 10 (Suppl. 1), A109. [CrossRef]

32. Brown, R.; Madelin, G.; Lattanzi, R.; Chang, G.; Regatte, R.R.; Sodickson, D.K.; Wiggins, G.C. Design of a Nested Eight-Channel Sodium and Four-Channel Proton Coil for 7T Knee Imaging. Magn. Reson. Med. 2013, 70, 259-268. [CrossRef] [PubMed]

33. Graessl, A.; Ruehl, A.; Waiczies, H.; Resetar, A.; Hoffmann, S.H.; Rieger, J.; Wetterling, F.; Winter, L.; Nagel, A.M.; Niendorf, T. Sodium MRI of the human heart at 7.0 T: Preliminary results. NMR Biomed. 2015, 28, 967-975. [CrossRef] [PubMed]

34. James, J.R.; Lin, C.; Stark, H.; Dale, B.M.; Bansal, N. Optimization and Characterization of Sodium MRI Using 8-channel ${ }^{23} \mathrm{Na}$ and 2-channel ${ }^{1} \mathrm{H}$ RX/TX. In Proceedings of the 13th International Conference on Biomedical Engineering 2009 IFMBE Proceedings, Singapore, 3-6 December 2009; Lim, C.T., Goh, J.C.H., Eds.; Springer: Berlin/Heidelberg, Germany, 2009 ; Volume 23.

35. James, J.R.; Panda, A.; Lin, C.; Dydak, U.; Dale, B.M.; Bansa, N. In vivo sodium MR imaging of the abdomen at 3T. Abdom. Imaging 2015, 40, 2272-2280. [CrossRef]

36. Kim, J.-H.; Moon, C.M.; Park, B.-W.; Furlan, A.; Zhao, T.; Bae, K.T. Multichannel transceiver dual-tuned RF coil for proton/sodium MR imaging of knee cartilage at 3 T. Magn. Reson. Imaging 2012, 30, 562-571. [CrossRef] [PubMed]

37. Lakshmanan, K.; Brown, R.; Madelin, G.; Qian, Y.; Boada, F.; Wiggins, G.C. An eight-channel sodium/proton coil for brain MRI at 3T. NMR Biomed. 2018, 31, e3867. [CrossRef]

38. Malzacher, M.; Chacon-Caldera, J.; Paschke, N.; Schad, L.R. Feasibility study of a double resonant 8-channel ${ }^{1} \mathrm{H} / 8$-channel ${ }^{23} \mathrm{Na}$ receive-only head coil at 3 Tesla. Magn. Reson. Imaging 2019, 59, 97-104. [CrossRef]

39. Woessner, D.E. NMR relaxation of spin-3/2 nuclei: Effects of structure, order, and dynamics in aqueous heterogeneous systems. Conc. Magn. Reson. 2001, 13, 294-325. [CrossRef]

40. Boada, F.E.; Gillen, J.S.; Shen, G.X.; Chang, S.Y.; Thulborn, K.R. Fast three dimensional sodium imaging. Magn. Reson. Med. 1997, 37, 706-715. [CrossRef] [PubMed]

41. Bernstein, M.A.; King, K.F.; Zhou, X.J. Handbook of MRI Pulse Sequences; Elsevier: San Diego, CA, USA, 2004.

42. Riemer, F.; Solanky, B.S.; Stehning, C.; Clemence, M.; Wheeler-Kingshott, C.A.; Golay, X. Sodium (23Na) ultra-short echo time imaging in the human brain using a 3D-Cones trajectory. Magn. Reson. Mat. Phys. Biol. Med. 2014, 27, 35-46. [CrossRef]

43. Gai, N.D.; Rochitte, C.; Nacif, M.S.; Bluemke, D.A. Optimized three-dimensional sodium imaging of the human heart on a clinical 3T scanner. Magn. Reson. Med. 2015, 73, 623-632. [CrossRef] [PubMed]

44. Ouwerkerk, R.; Weiss, R.G.; Bottomley, P.A. Measuring human cardiac tissue sodium concentrations using surface coils, adiabatic excitation, and twisted projection imaging with minimal T2 losses. J. Magn. Reson. Imaging 2005, 21, 546-555. [CrossRef] [PubMed]

45. Lott, J.; Platt, T.; Niesporek, S.C.; Paech, D.; Behl, N.G.R.; Niendorf, T.; Bachert, P.; Ladd, M.E.; Nagel, A.M. Corrections of myocardial tissue sodium concentration measurements in human cardiac 23 Na MRI at 7 Tesla. Magn. Reson. Med. 2019, 82, 159-173. [CrossRef]

46. Ragab, M.; Omer, O.A.; Abdel-Nasser, M. Compressive sensing MRI reconstruction using empirical wavelet transform and grey wolf optimizer. Neural Comput. Appl. 2020, 32, 2705-2724. [CrossRef]

47. Berendsen, H.J.; Edzes, H.T. The observation and general interpretation of sodium magnetic resonance in biological material. Ann. N. Y. Acad. Sci. 1973, 204, 459-485. [CrossRef] [PubMed]

48. Madelin, G.; Lee, J.-S.; Regatte, R.R.; Jerschow, A. Sodium MRI: Methods and applications. Prog. Nucl. Magn. Reson. Spectrosc. 2014, 79, 14-47. [CrossRef] [PubMed]

49. Hubbard, P.S. Nonexponential nuclear magnetic relaxation by quadrupole interactions. J. Chem. Phys. 1970, 53, 985-987. [CrossRef]

50. Blunck, Y.; Josan, S.; Taqdees, S.W.; Moffat, B.A.; Ordidge, R.J.; Cleary, J.O.; Johnston, L.A. 3D-multi-echo radial imaging of ${ }^{23} \mathrm{Na}$ (3D-MERINA) for time-efficient multi-parameter tissue compartment mapping. Magn. Reson. Med. 2018, 79, $1950-1961$. [CrossRef]

51. Riemer, F.; Solanky, B.; Clemence, M.; Wheeler-Kingshott, C.A.; Golay, X. Bi-exponential ${ }^{23} \mathrm{Na}$ T2* components analysis in the human brain. In Proceedings of the 20th Annual Meeting ISMRM, Melbourne, Australia, 5-11 May 2012; Volume 31, p. e3899.

52. Syeda, W.; Blunck, Y.; Kolbe, S.; Cleary, J.O.; Johnston, L.A. A continuum of components: Flexible fast fraction mapping in sodium MRI. Magn. Reson. Med. 2019, 81, 3854-3864. [CrossRef] [PubMed]

53. Thulborn, K.R.; Gindin, T.S.; Davis, D.; Erb, P. Comprehensive MRI protocol for stroke management: Tissue sodium concentration as a measure of tissue viability in a non-human primate model and clinical studies. Radiology 1999, 139, $26-34$. 
54. Constantinides, C.D.; Gillen, J.S.; Boada, F.E.; Pomper, M.G.; Bottomley, P.A. Human skeletal muscle: Sodium MR imaging and quantification potential applications in exercise and disease. Radiology 2000, 216, 559-568. [CrossRef]

55. Bansal, N.; Szczepaniak, L.; Ternullo, D.; Fleckenstein, J.L.; Malloy, C.R. Effect of exercise on 23Na MRI and relaxation characteristics of the human calf muscle. J. Magn. Reson. Imaging 2000, 11, 532-538. [CrossRef]

56. Hillenbrand, H.B.; Becker, L.C.; Kharrazian, R.; Hu, K.; Rochitte, C.E.; Kim, R.J.; Chen, E.L.; Ertl, G.; Hruban, R.H.; Lima, J.A.C. $23 \mathrm{Na}$ MRI combined with contrast-enhanced $1 \mathrm{H}$ MRI provides in vivo characterization of infarct healing. Magn. Reson. Med. 2005, 53, 843-850. [CrossRef]

57. Horn, M.; Weidensteiner, C.; Scheffer, H.; Meininger, M.; de Groot, M.; Remkes, H.; Dienesch, C.; Przyklenk, K.; von Kienlin, M.; Neubauer, S. Detection of myocardial viability based on measurement of sodium content: A Na-23-NMR study. Magn. Reson. Med. 2001, 45, 756-764. [CrossRef]

58. Thulborn, K.R.; Lu, A.; Atkinson, I.C.; Damen, F.; Villano, J.L. Quantitative sodium MR imaging and sodium bioscales for the management of brain tumors. Neuroimaging Clin. N. Am. 2009, 19, 615-624. [CrossRef] [PubMed]

59. Ouwerkerk, R.; Bottomley, P.A.; Solaiyappan, M.; Spooner, A.E.; Tomaselli, G.F.; Wu, K.C.; Weiss, R.G. Tissue sodium concentration in myocardial infarction in humans: A quantitative 23Na MR imaging study. Radiology 2008, 248, 88-96. [CrossRef] [PubMed]

60. Kopp, C.; Linz, P.; Wachsmuth, L.; Dahlmann, A.; Horbach, T.; Schöfl, C.; Renz, W.; Santoro, D.; Niendorf, T.; Müller, D.N.; et al. 23Na magnetic resonance imaging of tissue sodium. Hypertension 2012, 59, 167-172. [CrossRef] [PubMed]

61. Kopp, C.; Linz, P.; Dahlmann, A.; Hammon, M.; Jantsch, J.; Muller, D.N.; Schmieder, R.E.; Cavallaro, A.; Eckardt, K.; Uder, M.; et al. 23Na magnetic resonance imaging determined tissue sodium in healthy subjects and hyoertensive patients. Hypertension 2013, 61, 635-640. [CrossRef] [PubMed]

62. Christa, M.; Weng, A.M.; Geier, B.; Wörmann, C.; Scheffler, A.; Lehmann, L.; Oberberger, J.; Kraus, B.J.; Hahner, S.; Störk, S.; et al. Increased myocardial sodium signal intensity in Conn's syndrome detected by 23Na magnetic resonance imaging. Eur. Heart J. Cardiovasc. Imaging 2019, 20, 263-270. [CrossRef]

63. Kannenkeril, D.; Karg, M.V.; Bosch, A.; Ott, C.; Linz, P.; Nagel, A.M.; Uder, M.; Schmieder, R.E. Tissue sodium content is increased in type 2 diabetes. Diabetologia 2017, 60, 1-608.

64. Karg, M.V.; Bosch, A.; Kannenkeril, D.; Striepe, K.; Ott, C.; Schneider, M.P.; Boemke-Zelch, F.; Linz, P.; Nagel, A.M.; Titze, J.; et al. SGLT-2 inhibition with dapagliflozin reduces tissue sodium content a randomised controlled trial. Cardiovasc. Diabetol. 2018, 17, 5. [CrossRef]

65. Dyke, J.P.; Meyring-Wösten, A.; Zhao, Y.; Linz, P.; Thijssen, S.; Kotanko, P. Reliability and agreement of sodium (23Na) MRI in calf muscle and skin of healthy subjects from the US. Clin. Imaging 2018, 52, 100-105. [CrossRef]

66. Sekino, S.Y.; Sekino, M.; Tatsuoka, H.; Ohsaki, H.; Ueno, S.; Abe, Y. Discriminative Detection of Extracellular and Intracellular Sodium in Nerve Fibers by Magnetic Resonance Spectroscopy. IEEE Trans. Magn. 2008, 44, 4500-4502. [CrossRef] 